\title{
Energy Dependence of the Differences between the Quark and Gluon Jet Fragmentation
}

\author{
DELPHI Collaboration
}

\begin{abstract}
Three jet events arising from decays of the $\mathrm{Z}$ boson, collected by the DELPHI detector, were used to measure differences in quark and gluon fragmentation. Gluon jets were antitagged by identifying $b$ quark jets. Unbiased quark jets came from events with two jets plus one photon. Quark and gluon jet properties in different energy ranges were compared for the first time within the same detector. Quark and gluon jets of nearly the same energy in symmetric three jet event topologies were also compared. Using three independent methods, the average value of the ratio of the mean charged multiplicities of gluon and quark jets is

$$
<r\rangle=1.241 \pm 0.015 \text { (stat.) } \pm 0.025 \text { (syst.). }
$$

Gluon jets are broader and produce fragments with a softer energy spectrum than quark jets of equivalent energy. The string effect has been observed in fully symmetric three jet events. The measured ratio $R_{\gamma}$ of the charged particle flow in the $q \bar{q}$ inter-jet region of the $q \bar{q} g$ and $q \bar{q} \gamma$ samples agrees with the perturbative QCD expectation.

The dependence of the mean charged multiplicity on the hadronic center-of-mass energy was analysed in photon plus $n$-jet events. The value for $\alpha_{s}\left(M_{Z}\right)$ determined from these data using a QCD prediction with corrections at leading and next-to-leading order is

$$
\left.\alpha_{s}\left(M_{Z}\right)=0.116 \pm 0.003 \text { (stat. }\right) \pm 0.009 \text { (syst.). }
$$
\end{abstract}

(To be submitted to Zeit f. Physik C.) 
P.Abreu ${ }^{21}$, W.Adam ${ }^{50}$, T.Adye ${ }^{37}$, E.Agasi ${ }^{31}$, I.Ajinenko ${ }^{42}$, R.Aleksan ${ }^{39}$, G.D.Alekseev ${ }^{16}$, R.Alemany ${ }^{49}$, P.P.Allport ${ }^{22}$, S.Almehed ${ }^{24}$, S.J.Alvsvaag ${ }^{4}$, U.Amaldi ${ }^{9}$, S.Amato ${ }^{47}$, A.Andreazza ${ }^{28}$, M.L.Andrieux ${ }^{14}$, P.Antilogus $^{9}$, W-D.Apel ${ }^{17}$, Y.Arnoud ${ }^{39}$, B. ssman $^{44}$, J-E.Augustin ${ }^{19}$, A.Augustinus ${ }^{31}$, P.Baillon ${ }^{9}$, P.Bambade $^{19}$, F.Barao $^{21}$, R.Barate ${ }^{14}$, D.Y.Bardin ${ }^{16}$, A.Baroncelli ${ }^{40}$, O.Barring ${ }^{24}$, J.A.Barrio ${ }^{26}$, W.Bartl ${ }^{50}$, M.J.Bates ${ }^{37}$, M.Battaglia ${ }^{15}$, M.Baubillier ${ }^{23}$, J.Baudot ${ }^{39}$, K-H.Becks ${ }^{52}$, M.Begalli ${ }^{6}$, P.Beilliere ${ }^{8}$, Yu.Belokopytov ${ }^{9,53}$, K.Belous $^{42}$, A.C.Benvenuti ${ }^{5}$, M.Berggren ${ }^{47}$, D.Bertrand ${ }^{2}$, F.Bianchi ${ }^{45}$, M.Bigi ${ }^{45}$, M.S.Bilenky ${ }^{16}$, P.Billoir ${ }^{23}$, D.Bloch $^{10}$, M.Blume ${ }^{52}$, S.Blyth ${ }^{35}$, T.Bolognese ${ }^{39}$, M.Bonesini ${ }^{28}$, W.Bonivento ${ }^{28}$, P.S.L.Booth ${ }^{22}$, G.Borisov ${ }^{42}$, C.Bosio $^{40}$, S.Bosworth ${ }^{35}$, O.Botner ${ }^{48}$, B.Bouquet ${ }^{19}$, C.Bourdarios ${ }^{9}$, T.J.V.Bowcock ${ }^{22}$, M.Bozzo ${ }^{13}$, P.Branchini $^{40}$, K.D.Brand ${ }^{36}$, T.Brenke ${ }^{52}$, R.A.Brenner ${ }^{15}$, C.Bricman ${ }^{2}$, L.Brillault ${ }^{23}$, R.C.A.Brown ${ }^{9}$, P.Bruckman $^{18}$, J-M.Brunet ${ }^{8}$, L.Bugge ${ }^{33}$, T.Buran ${ }^{33}$, T.Burgsmueller ${ }^{52}$, P.Buschmann ${ }^{52}$, A.Buys ${ }^{9}$, S.Cabrera ${ }^{49}$, M.Caccia ${ }^{28}$, M.Calvi $^{28}$, A.J.Camacho Rozas ${ }^{41}$, T.Camporesi ${ }^{9}$, V.Canale ${ }^{38}$, M.Canepa ${ }^{13}$, K.Cankocak $^{44}$, F.Cao $^{2}$, F.Carena ${ }^{9}$, P.Carrilho ${ }^{47}$, L.Carroll ${ }^{22}$, C.Caso $^{13}$, M.V.Castillo Gimenez ${ }^{49}$, A.Cattai ${ }^{9}$, F.R.Cavallo ${ }^{5}$, L.Cerrito $^{38}{ }^{3}$, V.Chabaud ${ }^{9}$, Ph.Charpentier ${ }^{9}$, L.Chaussard ${ }^{25}$, J.Chauveau ${ }^{23}$, P.Checchia ${ }^{36}$, G.A.Chelkov ${ }^{16}$, M.Chen ${ }^{2}$, R.Chierici $^{45}$, P.Chliapnikov ${ }^{42}$, P.Chochula ${ }^{7}$, V.Chorowicz ${ }^{9}$, J.Chudoba ${ }^{30}$, V.Cindro ${ }^{43}$, P.Collins ${ }^{9}$, J.L.Contreras ${ }^{19}$, R.Contri ${ }^{13}$, E.Cortina ${ }^{49}$, G.Cosme ${ }^{19}$, F.Cossutti ${ }^{46}$, H.B.Crawley ${ }^{1}$, D.Crennell ${ }^{37}$, G.Crosetti $^{13}$, J.Cuevas Maestro $^{34}$, S.Czellar ${ }^{15}$, E.Dahl-Jensen ${ }^{29}$, J.Dahm ${ }^{52}$, B.Dalmagne ${ }^{19}$, M.Dam ${ }^{29}$, G.Damgaard ${ }^{29}$, P.D.Dauncey ${ }^{37}$, M.Davenport ${ }^{9}$, W.Da Silva ${ }^{23}$, C.Defoix ${ }^{8}$, A.Deghorain ${ }^{2}$, G.Della Ricca ${ }^{46}$, P.Delpierre ${ }^{27}$, N.Demaria $^{35}$, A.De Angelis ${ }^{9}$, W.De Boer ${ }^{17}$, S.De Brabandere ${ }^{2}$, C.De Clercq ${ }^{2}$, C.De La Vaissiere ${ }^{23}$, B.De Lotto ${ }^{46}$, A.De Min ${ }^{36}$, L.De Paula ${ }^{47}$, C.De Saint-Jean ${ }^{39}$, H.Dijkstra ${ }^{9}$, L.Di Ciaccio ${ }^{38}$, F.Djama $^{10}$, J.Dolbeau $^{8}$, M.Donszelmann ${ }^{9}$, K.Doroba ${ }^{51}$, M.Dracos ${ }^{10}$, J.Drees ${ }^{52}$, K.-A.Drees ${ }^{52}$, M.Dris ${ }^{32}$, Y.Dufour ${ }^{9}$, F.Dupont ${ }^{14}$, D.Edsall ${ }^{1}$, R.Ehret ${ }^{17}$, G.Eigen ${ }^{4}$, T.Ekelof ${ }^{48}$, G.Ekspong ${ }^{44}$, M.Elsing ${ }^{52}$, J-P.Engel ${ }^{10}$, N.Ershaidat ${ }^{23}{ }^{\text {, }}$, B.Erzen $^{43}$, M.Espirito Santo ${ }^{21}$, E.Falk ${ }^{24}$, D.Fassouliotis ${ }^{32}$, M.Feindt ${ }^{9}$, A.Ferrer ${ }^{49}$, T.A.Filippas ${ }^{32}$, A.Firestone ${ }^{1}$, P.-A.Fischer ${ }^{10}$, H.Foeth ${ }^{9}$, E.Fokitis ${ }^{32}$, F.Fontanelli ${ }^{13}$, F.Formenti ${ }^{9}$, B.Franek ${ }^{37}$, P.Frenkiel ${ }^{8}$, D.C.Fries ${ }^{17}$, A.G.Frodesen ${ }^{4}$, R.Fruhwirth ${ }^{50}$, F.Fulda-Quenzer ${ }^{19}$, J.Fuster ${ }^{49}$, A.Galloni ${ }^{22}$, D.Gamba ${ }^{45}$, M.Gandelman ${ }^{6}$, C.Garcia $^{49}$, J.Garcia ${ }^{41}$, C.Gaspar ${ }^{9}$, U.Gasparini ${ }^{36}$, Ph.Gavillet ${ }^{9}$, E.N.Gazis ${ }^{32}$, D.Gele ${ }^{10}$, J-P.Gerber $^{10}$, L.Gerdyukov ${ }^{42}$, M.Gibbs ${ }^{22}$, R.Gokieli ${ }^{51}$, B.Golob ${ }^{43}$, G.Gopal ${ }^{37}$, L.Gorn ${ }^{1}$, M.Gorski ${ }^{51}$, Yu.Gouz ${ }^{45,53}$, V.Gracco $^{13}$, E.Graziani ${ }^{40}$, G.Grosdidier ${ }^{19}$, K.Grzelak ${ }^{51}$, S.Gumenyuk ${ }^{28,53}$, P.Gunnarsson ${ }^{44}$, M.Gunther ${ }^{48}$, J.Guy $^{37}$, F.Hahn ${ }^{9}$, S.Hahn ${ }^{52}$, A.Hallgren ${ }^{48}$, K.Hamacher ${ }^{52}$, W.Hao ${ }^{31}$, F.J.Harris ${ }^{35}$, V.Hedberg ${ }^{24}$, R.Henriques $^{21}$, J.J.Hernandez ${ }^{49}$, P.Herquet ${ }^{2}$, H.Herr ${ }^{9}$, T.L.Hessing ${ }^{35}$, E.Higon ${ }^{49}$, H.J.Hilke ${ }^{9}$, T.S.Hill ${ }^{1}$, S-O.Holmgren ${ }^{44}$, P.J.Holt ${ }^{35}$, D.Holthuizen ${ }^{31}$, S.Hoorelbeke ${ }^{2}$, M.Houlden ${ }^{22}$, J.Hrubec ${ }^{50}$, K.Huet ${ }^{2}$, K.Hultqvist ${ }^{44}$, J.N.Jackson ${ }^{22}$, R.Jacobsson ${ }^{44}$, P.Jalocha ${ }^{18}$, R.Janik ${ }^{7}$, Ch.Jarlskog ${ }^{24}$, G.Jarlskog ${ }^{24}$, P.Jarry ${ }^{39}$, B.Jean-Marie ${ }^{19}$, E.K.Johansson ${ }^{44}$, L.Jonsson ${ }^{24}$, P.Jonsson ${ }^{24}$, C.Joram ${ }^{9}$, P.Juillot ${ }^{10}$, M.Kaiser ${ }^{17}$, F.Kapusta ${ }^{23}$, K.Karafasoulis ${ }^{11}$, M.Karlsson ${ }^{44}$, E.Karvelas ${ }^{11}$, S.Katsanevas ${ }^{3}$, E.C.Katsoufis ${ }^{32}$, R.Keranen ${ }^{44}$, B.A.Khomenko ${ }^{16}$, N.N.Khovanski ${ }^{16}{ }{ }$, B.King ${ }^{22}$, N.J.Kjaer ${ }^{29}$, O.Klapp ${ }^{52}$, H.Klein ${ }^{9}$, A.Klovning ${ }^{4}$, P.Kluit ${ }^{31}$, B.Koene ${ }^{31}$, P.Kokkinias ${ }^{11}$, M.Koratzinos $^{9}$, K.Korcyl $^{18}$, V.Kostioukhine ${ }^{42}$, C.Kourkoumelis ${ }^{3}$, O.Kouznetsov ${ }^{13}$, P.-H.Kramer ${ }^{52}$, M.Krammer ${ }^{50}$, C.Kreuter $^{17}$, I.Kronkvist $^{24}$, Z.Krumstein ${ }^{16}$, W.Krupinski ${ }^{18}$, P.Kubinec ${ }^{7}$, W.Kucewicz ${ }^{18}$, K.Kurvinen ${ }^{15}$, C.Lacasta $^{49}$, I.Laktineh $^{25}$, S.Lamblot ${ }^{23}$, J.W.Lamsa ${ }^{1}$, L.Lanceri ${ }^{46}$, D.W.Lane ${ }^{1}$, P.Langefeld ${ }^{52}$, V.Lapin ${ }^{42}$, I.Last $^{22}{ }^{2}$, J-P.Laugier $^{39}$, R.Lauhakangas ${ }^{15}$, G.Leder ${ }^{50}$, F.Ledroit ${ }^{14}$, V.Lefebure ${ }^{2}$, C.K.Legan ${ }^{1}$, R.Leitner ${ }^{30}$, Y.Lemoigne $^{39}$, J.Lemonne $^{2}$, G.Lenzen ${ }^{52}$, V.Lepeltier ${ }^{19}$, T.Lesiak ${ }^{36}$, R.Lindner ${ }^{52}$, A.Lipniacka ${ }^{36}$, I.Lippi ${ }^{36}$, B.Loerstad $^{24}$, J.G.Loken ${ }^{35}$, J.M.Lopez ${ }^{41}$, M.A.Lopez Aguera ${ }^{41}$, D.Loukas ${ }^{11}$, P.Lutz ${ }^{39}$, L.Lyons ${ }^{35}$, J.MacNaughton ${ }^{50}$, G.Maehlum $^{17}$, V.Malychev ${ }^{16}$, F.Mand ${ }^{50}$, J.Marco ${ }^{41}$, B.Marechal ${ }^{47}$, M.Margoni ${ }^{36}$, J-C.Marin ${ }^{9}$, C.Mariotti ${ }^{40}$, A.Markou $^{11}$, T.Maron ${ }^{52}$, C.Martinez-Rivero ${ }^{41}$, F.Martinez-Vidal ${ }^{49}$, S.Marti i Garcia ${ }^{49}$, J.Masik ${ }^{30}$, F.Matorras $^{41}$, C.Matteuzzi ${ }^{9}$, G.Matthiae ${ }^{38}$, M.Mazzucato ${ }^{36}$, M.Mc Cubbin ${ }^{9}$, R.Mc Kay ${ }^{1}$, R.Mc Nulty ${ }^{22}$, J.Medbo $^{48}$, C.Meroni $^{28}$, S.Meyer ${ }^{17}$, W.T.Meyer ${ }^{1}$, M.Michelotto ${ }^{36}$, E.Migliore ${ }^{45}$, L.Mirabito $^{25}$, W.A.Mitaroff ${ }^{50}$, U.Mjoernmark $^{24}$, T.Moa ${ }^{44}$, R.Moeller ${ }^{29}$, K.Moenig ${ }^{9}$, M.R.Monge ${ }^{13}$, P.Morettini ${ }^{13}$, H.Mueller ${ }^{17}$, L.M.Mundim ${ }^{6}$, W.J.Murray $^{37}$, B.Muryn ${ }^{18}$, G.Myatt ${ }^{35}$, F.Naraghi ${ }^{14}$, F.L.Navarria ${ }^{5}$, S.Navas ${ }^{49}$, K.Nawrocki ${ }^{51}$, P.Negri ${ }^{28}$, W.Neumann ${ }^{52}$, N.Neumeister ${ }^{50}$, R.Nicolaidou ${ }^{3}$, B.S.Nielsen ${ }^{29}$, M.Nieuwenhuizen ${ }^{31}$, V.Nikolaenko ${ }^{10}$, P.Niss ${ }^{44}$, A.Nomerotski ${ }^{36}$, A.Normand ${ }^{35}$, W.Oberschulte-Beckmann ${ }^{17}$, V.Obraztsov ${ }^{42}$, A.G.Olshevski ${ }^{16}$, A.Onofre ${ }^{21}$, R.Orava $^{15}$, K.Osterberg ${ }^{15}$, A.Ouraou ${ }^{39}$, P.Paganini ${ }^{19}$, M.Paganoni ${ }^{9}$, P.Pages ${ }^{10}$, H.Palka ${ }^{18}$, Th.D.Papadopoulou ${ }^{32}$, K.Papageorgiou ${ }^{11}$, L.Pape ${ }^{9}$, C.Parkes ${ }^{35}$, F.Parodi ${ }^{13}$, A.Passeri ${ }^{40}$, M.Pegoraro $^{36}$, L.Peralta $^{21}$, H.Pernegger ${ }^{50}$, M.Pernicka ${ }^{50}$, A.Perrotta ${ }^{5}$, C.Petridou ${ }^{46}$, A.Petrolini ${ }^{13}$, M.Petrovyck ${ }^{28,53}$, H.T.Phillips ${ }^{37}$, G.Piana ${ }^{13}$, F.Pierre ${ }^{39}$, M.Pimenta ${ }^{21}$, M.Pindo ${ }^{28}$, S.Plaszczynski ${ }^{19}$, O.Podobrin ${ }^{17}$, M.E.Pol ${ }^{6}$, G.Polok $^{18}$, P.Poropat ${ }^{46}$, V.Pozdniakov ${ }^{16}$, M.Prest ${ }^{46}$, P.Privitera ${ }^{38}$, N.Pukhaeva ${ }^{16}$, A.Pullia ${ }^{28}$, D.Radojicic ${ }^{35}$, S.Ragazzi $^{28}$, H.Rahmani ${ }^{32}$, J.Rames ${ }^{12}$, P.N.Ratoff ${ }^{20}$, A.L.Read ${ }^{33}$, M.Reale ${ }^{52}$, P.Rebecchi ${ }^{19}$, N.G.Redaelli ${ }^{28}$, M.Regler $^{50}$, D.Reid ${ }^{9}$, P.B.Renton ${ }^{35}$, L.K.Resvanis ${ }^{3}$, F.Richard ${ }^{19}$, J.Richardson ${ }^{22}$, J.Ridky ${ }^{12}$, G.Rinaudo ${ }^{45}$, I.Ripp $^{39}$, A.Romero ${ }^{45}$, I.Roncagliolo ${ }^{13}$, P.Ronchese ${ }^{36}$, L.Roos ${ }^{14}$, E.I.Rosenberg ${ }^{1}$, E.Rosso ${ }^{9}$, P.Roudeau ${ }^{19}$, T.Rovelli $^{5}$, W.Ruckstuhl ${ }^{31}$, V.Ruhlmann-Kleider ${ }^{39}$, A.Ruiz ${ }^{41}$, H.Saarikko ${ }^{15}$, Y.Sacquin ${ }^{39}$, A.Sadovsky ${ }^{16}$, G.Sajot $^{14}$, J.Salt ${ }^{49}$, J.Sanchez ${ }^{26}$, M.Sannino ${ }^{13}$, M.Schimmelpfennig ${ }^{17}$, H.Schneider ${ }^{17}$, U.Schwickerath ${ }^{17}$, M.A.E.Schyns ${ }^{52}$, G.Sciolla ${ }^{45}$, F.Scuri ${ }^{46}$, P.Seager $^{20}$, Y.Sedykh ${ }^{16}$, A.M.Segar ${ }^{35}$, A.Seitz ${ }^{17}$, R.Sekulin $^{37}$, R.C.Shellard ${ }^{6}$, I.Siccama ${ }^{31}$, P.Siegrist ${ }^{39}$, S.Simonetti ${ }^{39}$, F.Simonetto ${ }^{36}$, A.N.Sisakian ${ }^{16}$, B.Sitar ${ }^{7}$, T.B.Skaali $^{33}$, G.Smadja $^{25}$, N.Smirnov ${ }^{42}$, O.Smirnova ${ }^{16}$, G.R.Smith ${ }^{37}$, R.Sosnowski ${ }^{51}$, D.Souza-Santos ${ }^{6}$, T.Spassov ${ }^{21}$, E.Spiriti ${ }^{40}$, P.Sponholz ${ }^{52}$, S.Squarcia ${ }^{13}$, C.Stanescu ${ }^{40}$, S.Stapnes ${ }^{33}$, I.Stavitski ${ }^{36}$, F.Stichelbaut ${ }^{9}$, A.Stocchi ${ }^{19}$, J.Strauss $^{50}$, R.Strub ${ }^{10}$, B.Stugu ${ }^{4}$, M.Szczekowski ${ }^{51}$, M.Szeptycka ${ }^{51}$, T.Tabarelli ${ }^{28}$, J.P.Tavernet ${ }^{23}$, 
O.Tchikilev ${ }^{42}$, A.Tilquin ${ }^{27}$, J.Timmermans ${ }^{31}$, L.G.Tkatchev ${ }^{16}$, T.Todorov ${ }^{10}$, D.Z.Toet ${ }^{31}$, A.Tomaradze ${ }^{2}$, B. Tome ${ }^{21}$, A.Tonazzo ${ }^{28}$, L.Tortora ${ }^{40}$, G.Transtromer ${ }^{24}$, D.Treille ${ }^{9}$, W.Trischuk ${ }^{9}$, G.Tristram ${ }^{8}$, A.Trombini ${ }^{19}$, C.Troncon $^{28}$, A.Tsirou ${ }^{9}$, M-L.Turluer ${ }^{39}$, I.A.Tyapkin ${ }^{16}$, M.Tyndel ${ }^{37}$, S.Tzamarias ${ }^{22}$, B.Ueberschaer ${ }^{52}$, O.Ullaland ${ }^{9}$, V.Uvarov ${ }^{42}$, G.Valenti ${ }^{5}$, E.Vallazza ${ }^{9}$, C.Vander Velde ${ }^{2}$, G.W.Van Apeldoorn ${ }^{31}$, P.Van Dam ${ }^{31}$, W.K.Van Doninck ${ }^{2}$, J.Van Eldik ${ }^{31}$, N.Vassilopoulos ${ }^{35}$, G.Vegni ${ }^{28}$, L.Ventura ${ }^{36}$, W.Venus ${ }^{37}$, F.Verbeure ${ }^{2}$, M.Verlato $^{36}$, L.S.Vertogradov ${ }^{16}$, D.Vilanova ${ }^{39}$, P.Vincent ${ }^{25}$, L.Vitale ${ }^{46}$, E.Vlasov ${ }^{42}$, A.S.Vodopyanov $^{16}$, V.Vrba $^{12}$, H.Wahlen ${ }^{52}$, C.Walck ${ }^{44}$, F.Waldner ${ }^{46}$, M.Weierstall ${ }^{52}$, P.Weilhammer ${ }^{9}$, C.Weiser ${ }^{17}$, A.M.Wetherell ${ }^{9}$, D.Wicke ${ }^{52}$, J.H.Wickens ${ }^{2}, \quad$ M.Wielers ${ }^{17}$, G.R.Wilkinson ${ }^{35}$, W.S.C.Williams ${ }^{35}, \quad$ M.Winter ${ }^{10}$, M.Witek $^{18}$, K.Woschnagg $^{48}$, K.Yip ${ }^{35}$, O.Yushchenko ${ }^{42}$, F.Zach ${ }^{25}$, A.Zaitsev ${ }^{42}$, A.Zalewska ${ }^{18}$, P.Zalewski ${ }^{51}$, D.Zavrtanik ${ }^{43}$, E.Zevgolatakos ${ }^{11}$, N.I.Zimin ${ }^{16}$, M.Zito ${ }^{39}$, D.Zontar ${ }^{43}$, R.Zuberi ${ }^{35}$, G.C.Zucchelli ${ }^{44}$, G.Zumerle ${ }^{36}$

\footnotetext{
${ }^{1}$ Ames Laboratory and Department of Physics, Iowa State University, Ames IA 50011, USA

${ }^{2}$ Physics Department, Univ. Instelling Antwerpen, Universiteitsplein 1, B-2610 Wilrijk, Belgium and IIHE, ULB-VUB, Pleinlaan 2, B-1050 Brussels, Belgium

and Faculté des Sciences, Univ. de l'Etat Mons, Av. Maistriau 19, B-7000 Mons, Belgium

${ }^{3}$ Physics Laboratory, University of Athens, Solonos Str. 104, GR-10680 Athens, Greece

${ }^{4}$ Department of Physics, University of Bergen, Allégaten 55, N-5007 Bergen, Norway

${ }^{5}$ Dipartimento di Fisica, Università di Bologna and INFN, Via Irnerio 46, I-40126 Bologna, Italy

${ }^{6}$ Centro Brasileiro de Pesquisas Físicas, rua Xavier Sigaud 150, RJ-22290 Rio de Janeiro, Brazil and Depto. de Física, Pont. Univ. Católica, C.P. 38071 RJ-22453 Rio de Janeiro, Brazil and Inst. de Física, Univ. Estadual do Rio de Janeiro, rua São Francisco Xavier 524, Rio de Janeiro, Brazil ${ }^{7}$ Comenius University, Faculty of Mathematics and Physics, Mlynska Dolina, SK-84215 Bratislava, Slovakia

${ }^{8}$ Collège de France, Lab. de Physique Corpusculaire, IN2P3-CNRS, F-75231 Paris Cedex 05, France

${ }^{9} \mathrm{CERN}, \mathrm{CH}-1211$ Geneva 23, Switzerland

${ }^{10}$ Centre de Recherche Nucléaire, IN2P3 - CNRS/ULP - BP20, F-67037 Strasbourg Cedex, France

${ }^{11}$ Institute of Nuclear Physics, N.C.S.R. Demokritos, P.O. Box 60228, GR-15310 Athens, Greece

${ }^{12}$ FZU, Inst. of Physics of the C.A.S. High Energy Physics Division, Na Slovance 2, 180 40, Praha 8, Czech Republic

${ }^{13}$ Dipartimento di Fisica, Università di Genova and INFN, Via Dodecaneso 33, I-16146 Genova, Italy

${ }^{14}$ Institut des Sciences Nucléaires, IN2P3-CNRS, Université de Grenoble 1, F-38026 Grenoble Cedex, France

${ }^{15}$ Research Institute for High Energy Physics, SEFT, P.O. Box 9, FIN-00014 Helsinki, Finland

${ }^{16}$ Joint Institute for Nuclear Research, Dubna, Head Post Office, P.O. Box 79, 101000 Moscow, Russian Federation

${ }^{17}$ Institut für Experimentelle Kernphysik, Universität Karlsruhe, Postfach 6980, D-76128 Karlsruhe, Germany

${ }^{18}$ Institute of Nuclear Physics and University of Mining and Metalurgy, Ul. Kawiory 26a, PL-30055 Krakow, Poland

${ }^{19}$ Université de Paris-Sud, Lab. de l'Accélérateur Linéaire, IN2P3-CNRS, Bât. 200, F-91405 Orsay Cedex, France

${ }^{20}$ School of Physics and Materials, University of Lancaster, Lancaster LA1 4YB, UK

${ }^{21}$ LIP, IST, FCUL - Av. Elias Garcia, 14-1 ${ }^{\circ}$, P-1000 Lisboa Codex, Portugal

${ }^{22}$ Department of Physics, University of Liverpool, P.O. Box 147, Liverpool L69 3BX, UK

${ }^{23}$ LPNHE, IN2P3-CNRS, Universités Paris VI et VII, Tour 33 (RdC), 4 place Jussieu, F-75252 Paris Cedex 05, France

${ }^{24}$ Department of Physics, University of Lund, Sölvegatan 14, S-22363 Lund, Sweden

${ }^{25}$ Université Claude Bernard de Lyon, IPNL, IN2P3-CNRS, F-69622 Villeurbanne Cedex, France

${ }^{26}$ Universidad Complutense, Avda. Complutense s/n, E-28040 Madrid, Spain

${ }^{27}$ Univ. d'Aix - Marseille II - CPP, IN2P3-CNRS, F-13288 Marseille Cedex 09, France

${ }^{28}$ Dipartimento di Fisica, Università di Milano and INFN, Via Celoria 16, I-20133 Milan, Italy

${ }^{29}$ Niels Bohr Institute, Blegdamsvej 17, DK-2100 Copenhagen 0, Denmark

${ }^{30} \mathrm{NC}$, Nuclear Centre of MFF, Charles University, Areal MFF, V Holesovickach 2, 180 00, Praha 8, Czech Republic

${ }^{31}$ NIKHEF-H, Postbus 41882, NL-1009 DB Amsterdam, The Netherlands

${ }^{32}$ National Technical University, Physics Department, Zografou Campus, GR-15773 Athens, Greece

${ }^{33}$ Physics Department, University of Oslo, Blindern, N-1000 Oslo 3, Norway

${ }^{34}$ Dpto. Fisica, Univ. Oviedo, C/P. Pérez Casas, S/N-33006 Oviedo, Spain

${ }^{35}$ Department of Physics, University of Oxford, Keble Road, Oxford OX1 3RH, UK

${ }^{36}$ Dipartimento di Fisica, Università di Padova and INFN, Via Marzolo 8, I-35131 Padua, Italy

${ }^{37}$ Rutherford Appleton Laboratory, Chilton, Didcot OX11 OQX, UK

${ }^{38}$ Dipartimento di Fisica, Università di Roma II and INFN, Tor Vergata, I-00173 Rome, Italy

${ }^{39}$ Centre d'Etudes de Saclay, DSM/DAPNIA, F-91191 Gif-sur-Yvette Cedex, France

${ }^{40}$ Istituto Superiore di Sanità, Ist. Naz. di Fisica Nucl. (INFN), Viale Regina Elena 299, I-00161 Rome, Italy

${ }^{41}$ Instituto de Fisica de Cantabria (CSIC-UC), Avda. los Castros, S/N-39006 Santander, Spain, (CICYT-AEN93-0832)

${ }^{42}$ Inst. for High Energy Physics, Serpukov P.O. Box 35, Protvino, (Moscow Region), Russian Federation

${ }^{43} \mathrm{~J}$. Stefan Institute and Department of Physics, University of Ljubljana, Jamova 39, SI-61000 Ljubljana, Slovenia

${ }^{44}$ Fysikum, Stockholm University, Box 6730, S-113 85 Stockholm, Sweden

${ }^{45}$ Dipartimento di Fisica Sperimentale, Università di Torino and INFN, Via P. Giuria 1, I-10125 Turin, Italy

${ }^{46}$ Dipartimento di Fisica, Università di Trieste and INFN, Via A. Valerio 2, I-34127 Trieste, Italy and Istituto di Fisica, Università di Udine, I-33100 Udine, Italy

${ }^{47}$ Univ. Federal do Rio de Janeiro, C.P. 68528 Cidade Univ., Ilha do Fundão BR-21945-970 Rio de Janeiro, Brazil

${ }^{48}$ Department of Radiation Sciences, University of Uppsala, P.O. Box 535, S-751 21 Uppsala, Sweden

${ }^{49}$ IFIC, Valencia-CSIC, and D.F.A.M.N., U. de Valencia, Avda. Dr. Moliner 50, E-46100 Burjassot (Valencia), Spain

${ }^{50}$ Institut für Hochenergiephysik, Österr. Akad. d. Wissensch., Nikolsdorfergasse 18, A-1050 Vienna, Austria

${ }^{51}$ Inst. Nuclear Studies and University of Warsaw, Ul. Hoza 69, PL-00681 Warsaw, Poland

${ }^{52}$ Fachbereich Physik, University of Wuppertal, Postfach 100 127, D-42097 Wuppertal 1, Germany

${ }^{53}$ On leave of absence from IHEP Serpukhov
} 


\section{Introduction}

In Quantum Chromodynamics (QCD) quarks and gluons are predicted to carry distinct colour charges. Quarks have a single colour index while gluons are tensor objects carrying two colour indices. Due to this fact, quarks and gluons differ in their relative coupling strength to emit additional gluons, and, in consequence, jets originating from the fragmentation of energetic quarks and gluons are expected to show differences in their final particle multiplicities, energies and angular distributions. The investigation of these differences is the subject of this article.

Earlier results of the study of differences between quark and gluon jets [1] at center of mass energies below the $Z$ mass indicated differences in the momentum and transverse momentum spectra of particles from quark and gluon jets. Only recently with the massive statistics and improved quark jet tagging techniques available at LEP have conclusive measurements of the multiplicity difference of quark and gluon jets become available [2,3].

Less than $1 \%$ of the gluon jets are expected to contain particles originating from the fragmentation of heavy $b$ quarks [4]. Gluon jets can therefore be collected from an initial sample of reconstructed three jet events, $q \bar{q} g$, in which two of the jets, the quark jets, are seen to satisfy the experimental signatures of being initiated by $b$ quarks, leaving the remaining jet to be associated to the gluon jet without further requirements. In the present analysis, the use of advanced experimental techniques to identify the original flavour of the quark jets with very high precision enables high gluon jet purities $(\sim 94 \%)$ to be attained, allowing thus a study of an almost background free sample of gluon jets. The variety of methods investigated to select the $b$ quark initiated jets includes the identification of inclusive high momentum leptons and the use of impact parameter distributions. The combination and comparison of all these methods acts as an important cross-check of the final results since they are subject to different systematic biases.

A further important ingredient of the analysis is the use of hadronic events containing two hadronic jets and an energetic, isolated photon. The selection of such $q \bar{q} \gamma$ events provides a sample of high purity quark jets of varying energy. Hence, for the first time, a direct comparison of quark and gluon jets, as a function of energy, can be performed within the same detector. The use of $q \bar{q} \gamma$ events can also be extended to the study of the string effect [5,6], which predicts a greater particle flow in the inter-jet region between the two quarks of the $q \bar{q} \gamma$ event types than in the corresponding analogous region of the $q \bar{q} g$ events $[7-10]$.

The large sample of hadronic events also allows the investigation of special symmetric event topologies to compare quark and gluon jets at nearly the same energy scale. Two types of symmetric three jet event topologies are studied in detail, two fold symmetric events and fully symmetric events. For the first time an analysis of fully symmetric events is presented in which both $b$ jets are tagged simultaneously.

The mean charged multiplicity of events containing one photon and any number of jets is also studied as a function of the reduced center-of-mass energy of the hadronic system. The comparison of the obtained distribution with results from other $e^{+} e^{-}$experiments at lower center-of-mass energies provides an interesting cross-check of the possible biases present in the quark jet sample. 


\section{Experimental Apparatus and Event Selection}

\subsection{The DELPHI Detector}

The DELPHI detector, surrounding one of the interaction regions at the Large Electron Positron facility LEP at CERN, has been used to record the samples of events contained in this analysis. It provides both tracking and calorimetric information over almost the full solid angle. A detailed description of the detector, the exact geometry as well as the trigger conditions and the event processing chain appear in references [11-13].

The barrel region of the detector consists of a system of cylindrical tracking detectors and an electromagnetic calorimeter, embedded in a superconducting solenoidal coil providing a uniform magnetic field of $1.23 \mathrm{~T}$ parallel to the beam direction $(z)$. The central tracking detectors provide measurements of the coordinates of charged particles in both the $R \Phi$ plane, transverse to the beam, and in the $z$ direction. These are the vertex detector, the inner detector, the time projection chamber and the outer detector. The vertex detector configuration comprises three concentric and overlapping layers of silicon microstrip detectors which allow the $(R, \Phi)$ coordinates of charged particles to be measured with a precision of $8 \mu \mathrm{m}$. The inner detector is a cylindrical jet chamber, providing $24(R, \Phi)$ coordinates, surrounded by an outer cylinder containing five layers of multiwire proportional chambers which give coordinates both in $(R, \Phi)$ and $z$ coordinates. The Time Projection Chamber (TPC) is the principal tracking device which in addition can provide a measurement of the energy loss $d E / d x$ for charged particles, with a resolution of $\pm 5.5 \%$ in muon pair events. The tracking in the barrel section is completed by the outer detector which is composed of five layers of drift cells. The combined usage of these tracking detectors provides an average momentum resolution of $\sigma(p) / p=3.6 \% \mathrm{GeV} / c$ for muons of $45 \mathrm{GeV} / c[14]$.

Electromagnetic calorimetry is accomplished in the barrel region by the High Density Projection Chamber (HPC), which covers polar angles $\theta$ from $40^{\circ}$ to $140^{\circ}$. The $\mathrm{HPC}$ is a gas sampling calorimeter, operating on the time-projection principle, which measures with high granularity the three-dimensional charge distribution induced by electromagnetic showers, enabling thus the identification of electrons and photons in a hadronic environment.

In each of the forward regions of the detector, two systems of drift chambers (FCA,FCB), covering polar angles between $11^{\circ}$ and $33^{\circ}$, improve the tracking of charged particles. The electromagnetic energy is measured by the Forward Electro Magnetic Calorimeter (FEMC), which consists of an array of lead glass blocks subtending polar angles from $8^{\circ}$ to $36^{\circ}$ on either side.

The muon detection system is both within and beyond the outer layers of the hadron calorimeter (HAC), which also serves as the return yoke of the magnet. In the barrel section, the system consists of several layers of drift chambers with delay line readout. In each of the forward regions, the system consists of two modules of drift chambers arranged in quadrants. In both the barrel and the forward regions, measurements of penetrating charged particles in three dimensions are provided.

\subsection{Event Selection}

All data collected by DELPHI during the years 1991 to 1993 were considered in the present analysis. In a first step of the selection procedure, quality cuts on all charged 


\begin{tabular}{|c|c|c|c||}
\hline Algorithm & Reference & Resolution & Recombination \\
\hline Durham $\left(k_{T}\right)$ & {$[17]$} & $y_{i j}=\frac{2 \cdot m i n\left(E_{i}^{2}, E_{j}^{2}\right) \cdot\left(1-\cos \theta_{i j}\right)}{E_{v i s}^{2}}$ & $p_{k}=p_{i}+p_{j}$ \\
JADE & {$[18]$} & $y_{i j}=\frac{\left(p_{i}+p_{j}\right)^{2}}{E_{v i s}^{2}}$ & $\begin{array}{c}\vec{p}_{k}=\frac{E_{k}}{\left|\vec{p}_{i}+\vec{p}_{j}\right|}\left(\vec{p}_{i}+\vec{p}_{j}\right) \\
E_{k}=E_{i}+E_{j}\end{array}$ \\
\hline
\end{tabular}

Table 1: Definition of the jet resolution variable $y_{i j}$ and of the recombination schemes for the DURHAM and JADE jet finding algorithms; $E_{\text {vis }}$ is the total visible energy of the event, $p_{i} \equiv\left(E_{i}, \vec{p}_{i}\right)$ denotes a 4 -vector and $\theta_{i j}$ is the angle between $\vec{p}_{i}$ and $\vec{p}_{j}$.

particles and all neutral clusters in the calorimeters were imposed in order to ensure a reliable determination of their momenta, energies and multiplicities. The quality cuts on charged particles were as in [15]; neutral clusters reconstructed in the HPC, FEMC and hadron calorimeter were selected by imposing requirements on the minimum and maximum reconstructed energy, with an additional condition on the distribution of layers hit for HPC clusters. Identified electron positron pairs arising from photon conversions were considered as single neutral clusters if the sum of their momenta exceeded 600 $\mathrm{MeV} / \mathrm{c}$.

A sample of hadronic events was then selected as in [15] by demanding a minimum charged multiplicity, enough visible charged energy and events well contained within the detector volume, with a veto on events containing badly mis-measured charged particles. Small differences in these cuts were used when studying particular topologies of events. The surviving data sample passing the hadronic criteria contained more than $1.6 \cdot 10^{6}$ events with a small contamination arising from $\tau^{+} \tau^{-}$pairs $(\sim 0.1 \%)$ and negligible contamination from beam-gas scattering and $\gamma \gamma$ interactions.

Charged and neutral particles were grouped into jets by means of a particular jet finding algorithm. The general procedure was as follows. For each pair of particles $i j$, the algorithm characteristic jet resolution variable $y_{i j}$ was calculated from the corresponding four-momentum vectors of both particles. The pair with the smallest $y_{i j}$ and whose value did not exceed a given threshold $y_{c u t}$, which determined the point at which particles were resolved into jets, was combined to form a new pseudo-particle with four-momentum as defined by a given recombination scheme. The procedure was reiterated until no further pairs of particles or pseudo-particles satisfied the condition $y_{i j}<y_{c u t}$. The remaining particles or pseudo-particles were henceforth referred to as jets.

A number of such jet finding algorithms have now been developed and their properties studied in detail [16]. The principal results of this analysis are presented using the DURHAM algorithm [17] and for comparison also the JADE algorithm [18]. They differ from one another in the definitions of the recombination scheme and of the jet resolution variable. Each has been applied to the hadronic data sample for the selection of three jet events and the assignment of particles to jets. As no jet finding algorithm can claim to be unique in the correct particle assignment to jets [19], the analysis of both selected 
three-jet data samples provides an important cross-check of the relevant results. Table 1 summarises the exact definition of the resolution variables and recombination schemes used by these two algorithms.

\subsection{Event Samples}

For a detailed comparison of quark and gluon jet properties it was necessary to obtain samples of quark and gluon jets with similar energies. Different event topologies were used to fulfil this condition as it is illustrated in figure 1.

a)

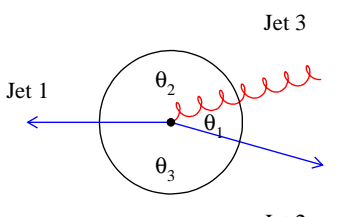

Jet 2

q⿳亠े冖 events b)

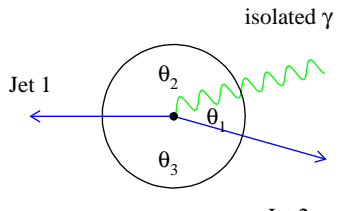

Jet 2

$\mathrm{q} \overline{\mathrm{q}} \gamma$ events

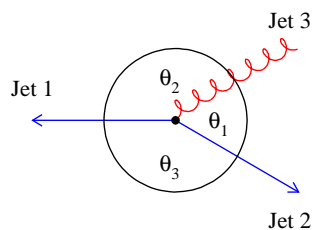

Y events; $\theta_{2,3} \in\left[150^{\circ} \pm 15^{\circ}\right]$

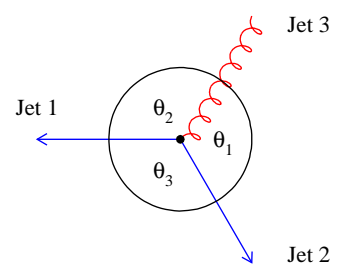

Mercedes events; $\theta_{2,3} \in\left[120^{\circ} \pm 15^{\circ}\right]$

Figure 1: Three jet event configurations of the gluon and quark jets analysed in the present study.

Three jet events, in which none of the jets consisted of an isolated photon, were used to select gluon jets (figure 1(a)). For each of these events, the two quark jets were recognised using experimental techniques which identified heavy quark initiated jets with high precision. The remaining jet was then assumed to originate from a gluon without any extra condition, therefore and henceforth referred to as anti-tagged. Events containing an isolated hard photon were used to obtain unbiased quark jets of reduced energy (figure $1(\mathrm{~b}))$. The properties of quark and gluon jets obtained in this way could thus be compared as a function of the jet energy. The quark sample was largely independent of any influence from hard gluon radiation, ensuring that systematic effects due to mixed quark and gluon samples were negligible. Unfortunately, the statistics obtained by this selection were rather low.

Symmetric events were selected by requiring $\theta_{2} \approx \theta_{3}$ ( $\theta$ being the jet-jet angle as in figure 1) defining one ( $\mathrm{Y}$ type events) or two quark jets (Mercedes type events) and one gluon jet of similar energy and topology (figures $1(\mathrm{c}, \mathrm{d})$ ). This nomenclature for twofold symmetric $(Y)$ events $\left(135^{\circ}<\theta_{2,3}<165^{\circ}\right)$ and threefold symmetric (Mercedes) events $\left(105^{\circ}<\theta_{2,3}<135^{\circ}\right)$ is used throughout this paper. For Mercedes events the gluon jets were obtained using the same technique as described above in which the two heavy quark jets were experimentally identified. For $\mathrm{Y}$ events, the most energetic jet was assumed to originate from a quark, which is true in $98 \%$ of the cases. The other two jets were then resolved to be one the quark and the other the gluon jet by searching for the heavy quark signature to be satisfied in only one of them. For these symmetric configurations, the gluon jets were directly compared to the mixed jet sample contained in all symmetric three jet events. The use of subtraction techniques which rely on the knowledge of the proportion of quark and gluon jets populating the three jet event sample enabled this comparison to be made. 
Note that none of the quark jets used to anti-tag gluon jets entered in the quarkgluon comparison since their topological properties were biased by the selection criteria. However, gluon jet properties remained unaltered by this procedure [20,21].

\subsection{1 $q \bar{q} g$ Event Sample}

In order to enhance the contribution from events with three well defined jets attributed to $q \bar{q} g$ production, a set of further cuts was applied to the three jet event samples. These cuts selected planar events with each of the reconstructed jets well contained within a detector region of good acceptance. The symmetric and non-symmetric configurations had separate cuts, summarised in table 2, to accommodate the different jet configurations and the statistics resulting from the sample selections.

\begin{tabular}{|l||c|c|}
\hline Measurement & Non-symmetric topologies & Symmetric topologies \\
\hline \hline Number of particles in each jet & $\geq 1$ (charged) & $\geq 2$ (charged or neutral) \\
\hline Minimum jet energy & $3 \mathrm{GeV}$ & $5 \mathrm{GeV}$ \\
\hline Sum of angles between jets & $>359.5^{\circ}$ & $>355^{\circ}$ \\
\hline Polar angle of each jet axis & $26^{\circ}-154^{\circ}$ & $30^{\circ}-150^{\circ}$ \\
\hline
\end{tabular}

Table 2: Planarity and acceptance cuts for reconstructed three jet events.

The values of $y_{\text {cut }}$ used for the different analyses when selecting the three jet data samples were optimised using the JeTseT 7.3 Parton Shower Monte Carlo [22] by maximising the available statistics and the purity of the three jet sample, and, minimising the fraction of four jet events in the three jet sample. The three jet purities were calculated by computing the fraction of three jet events reconstructed at both parton and hadron level w.r.t. all three jet events reconstructed after hadronisation. For events not restricted to have a symmetric configuration, values of $y_{c u t}=0.01$ for the DURHAM jet finder and $y_{c u t}=0.04$ for the JADE recombination scheme were the most suitable values, whereas a $y_{\text {cut }}=0.015$ was chosen to preselect the symmetric three jet events. When value of $y_{\text {cut }}=0.010$ was used, the sample of Mercedes events was primarily populated by $\mathrm{Y}$ events just passing the topological cuts, whereas many of the real Mercedes type like events were resolved as four jet events.

To each of the jets a calculated energy was assigned as derived from the jet directions and the angles between them. Assuming massless kinematics, the jet energy could be expressed as:

$$
E_{j}^{\text {calc }}=\frac{\sin \theta_{j}}{\sin \theta_{1}+\sin \theta_{2}+\sin \theta_{3}} \sqrt{s}, \quad j=1,2,3
$$

where $\theta_{j}$ is the inter-jet angle as defined in figure 1 .

Studies using full simulation of the DELPHI detector [23] showed that for the range of jet energies being considered here, from $5 \mathrm{GeV}$ up to $45 \mathrm{GeV}$, the calculated jet energy $E_{j}^{c a l c}$ gave a better representation of the true underlying jet energy $E_{j}^{\text {true }}$ (i.e. before detector simulation) than did the reconstructed (or visible) jet energy $E_{j}^{r e c}$. The use of Eq. (1) had in fact two effects. Primarily it corrected for the energy shift towards low values which affected the measured reconstructed jet energy due to undetected particles, 
and, secondly, improved the energy resolution from $\sigma\left(E_{j}^{r e c}-E_{j}^{\text {true }}\right) \approx 3.5-7.0 \mathrm{GeV}$ to $\sigma\left(E_{j}^{\text {calc }}-E_{j}^{\text {true }}\right) \approx 2.5-1.5 \mathrm{GeV}$ in this energy range.

Subsequent corrections to $E_{j}^{\text {calc }}$, due to the mass effects in the case of $b$ quark initiated jets, were also added to the above formula even though they only applied for small gluon energies and in all circumstances were small, less than $3 \%$.

Quark and gluon jets were then grouped in energy intervals of $5 \mathrm{GeV}$, covering the range from $7.5 \mathrm{GeV}$ up to $42.5 \mathrm{GeV}$, both in data and simulation data. The calculated jet energy, $E_{j}^{c a l c}$, was used to define the jet energy.

The symmetric topology event samples were not further divided into energy bins.

\subsection{2 $q \bar{q} \gamma$ Event Sample}

To enable a comparison of the selected gluon jets with an unbiased sample of quark jets of comparable energies, two jet events containing a hard radiative photon were selected.

Starting from the sample of events containing three jets, as determined by the particular jet-finding algorithm, the subset in which one of the jets was formed by only one neutral particle was subject to further analysis. This enhanced the contribution from events with a hard final state photon.

This enhancement was achieved by selecting only those photon candidates of energies greater than $5 \mathrm{GeV}$ that were deemed inconsistent with originating from a radiative electron. Photons recognised as converting into electron positron pairs were also considered in the selection criteria. It was further demanded that no charged particle was present within a cone of $20^{\circ}$ around the photon direction. The resulting isolation angle and the reconstructed energy spectrum of the selected photon candidates were observed to agree between the data and the simulation prediction [20]. Each of the jets was likewise assigned a calculated energy using Eq. 1, thereby minimising the sensitivity to possible discrepancies in the calibration of the detectors. Cases, where the calculated $E_{\gamma}^{\text {calc }}$ and the reconstructed $E_{\gamma}^{r e c}$ energies of the photon disagreed by more than $50 \%$ were seen to originate mainly from background processes and were therefore rejected.

For each event the information given by the remaining two quark jets was used in the analysis. Only two types of events contaminated the $q \bar{q} \gamma$ sample. These were hadronic final states with misidentified $\pi^{\circ}$ and radiative $\tau^{+} \tau^{-}$events. When the event selection used the DURHAM reconstruction algorithm the calculated backgrounds were $5.6 \%$ and $2.6 \%$ for misidentified $\pi^{\circ}$ and for radiative $\tau^{+} \tau^{-}$pairs, respectively.

The samples listed in table 3 were obtained after applying the quoted selection criteria.

\begin{tabular}{|c|c|c|c|}
\hline Event type & \# events & Energy range & $<E>$ \\
\hline $\begin{array}{ll}q \bar{q} g & (\text { DURHAM }) \\
(\mathrm{JADE})\end{array}$ & $\begin{array}{l}319,095 \\
328,355\end{array}$ & $2.5 \mathrm{GeV}-42.5 \mathrm{GeV}$ & 8 bins of $5 \mathrm{GeV}$ \\
\hline$q \bar{q} \gamma \quad \begin{array}{l}\text { (DURHAM) } \\
(\mathrm{JADE})\end{array}$ & $\begin{array}{l}1,006 \\
1,112\end{array}$ & $2.5 \mathrm{GeV}-42.5 \mathrm{GeV}$ & 8 bins of $5 \mathrm{GeV}$ \\
\hline Y events & 74,164 & & \\
\hline Mercedes events & 9,264 & $27.4 \mathrm{GeV}-33.4 \mathrm{GeV}$ & $30.4 \mathrm{GeV}$ \\
\hline
\end{tabular}

Table 3: The three jet event samples and their corresponding energy intervals as used in the present analysis. 


\section{Methods in Anti-tagging Gluon Jets in $q \bar{q} g$ Events}

In this section the different methods employed to extract the gluon induced jets in the selected samples of $q \bar{q} g$ events are introduced. The common approach of the various methods followed the general strategy of identifying the two quark jets using well known experimental techniques that efficiently detected the signature of heavy quark fragmentation. Gluon jets were thus selected by being the only jet not passing the heavy quark selection criteria in three jet events. The further advantage of this is that no special selection was directly required for the gluon jet and therefore biases were minimised.

\subsection{Lepton Identification}

Muons were identified by their ability to penetrate large amounts of material in the DELPHI detector. Muon candidates were first selected by requiring that particles detected by the tracking chambers penetrated the hadron calorimeter into the muon detector [24]. To discriminate against background from pion and kaon decays and hadron punch-through, a minimal momentum of $4 \mathrm{GeV} / c$ was demanded. Three jet events containing a muon candidate were then selected, but only those events, in which one of the two lower energy jets contained the lepton, were retained. The most energetic jet and the jet containing the lepton were thus tagged as quark jets, while the remaining third jet was considered to be the gluon jet. The total number of events thus selected were 8358 or 8462 using the DURHAM or JADE algorithm, respectively.

Electrons were identified by examining the response of the HPC to charged particles and by the energy loss, $d E / d x$, as measured in the TPC. A number of variables that described the longitudinal shower profiles were also constructed [24]. The combination of all this information together with the particle momentum $(\geq 3 \mathrm{GeV} / c)$ were then used to construct a single variable whose value returned the probability for the particle under consideration to be an electron. Electron candidates were thus selected by imposing tight cuts to this probability such that a high purity was achieved. The method of tagging (anti-tagging) the quark (gluon) jets described above was similarly applied to the electron inclusive three jet sample, giving a total of 7650 (DURHAM) or 7802 (JADE) gluon jet candidates.

\subsection{Lifetime Tag}

The lifetime signed impact parameter of charged particles was used to construct an algorithm for tagging $b$ jets following a method developed by the ALEPH Collaboration [25] which has recently been adapted to the DELPHI data [26]. In this method the probability $P_{N}$ to contain no decay products from long lived hadrons was evaluated for a given selection of $N$ particles. Each value of $P_{N}$ corresponded to a specific combination of $b$ purity and efficiency, which in the case of DELPHI appears in reference [26]. In this analysis, the whole sample of three jet events was considered and the tracks corresponding to each of the reconstructed jets were used to construct a probability $P_{J}$ per jet. Events and jets were finally classified according to the observed values of each $P_{J}$ following two selection strategies: 
Gluon jet energies
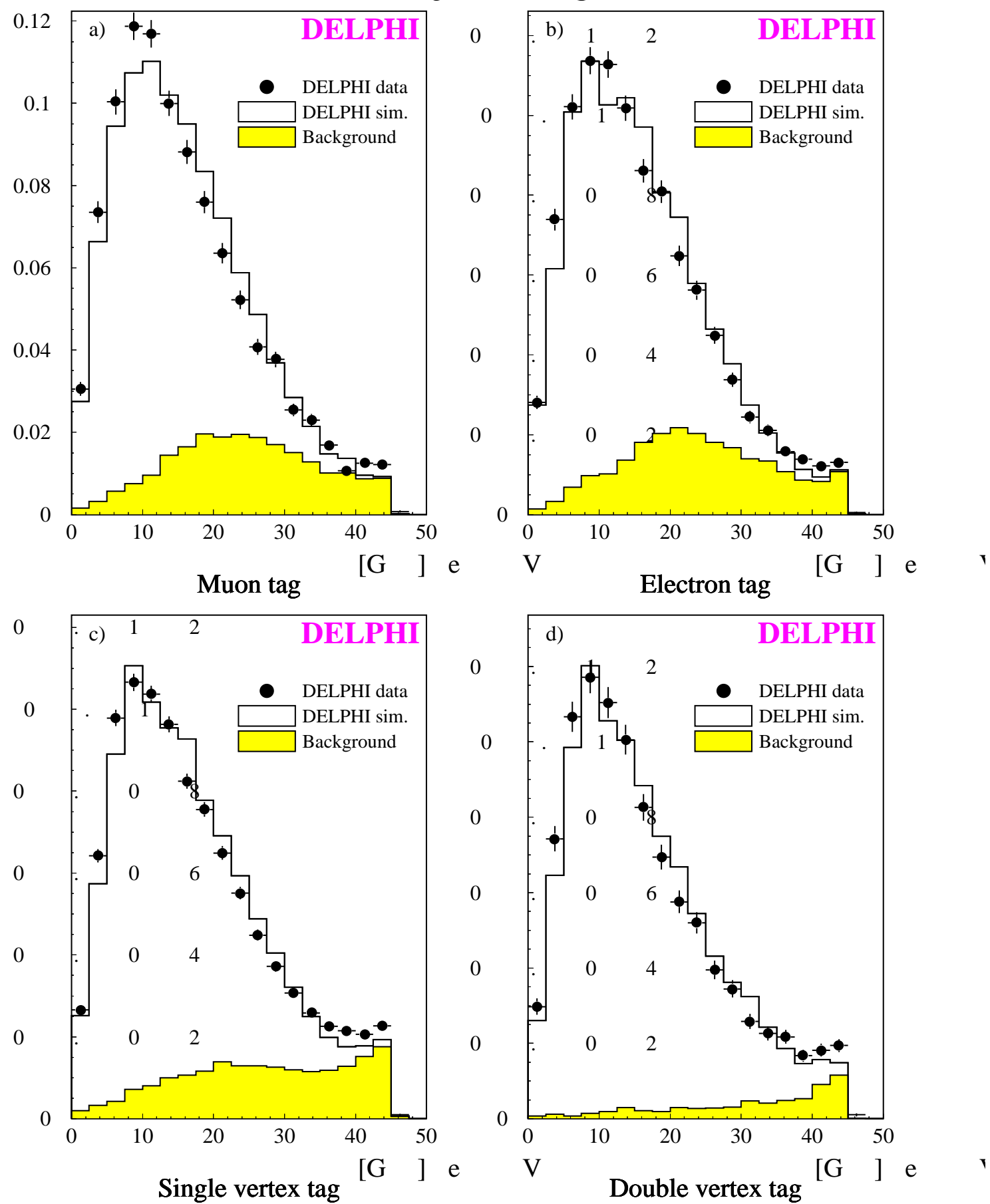

Figure 2: Normalised distributions of the gluon jet energy spectrum for the various samples as selected using (a) inclusive muons, (b) inclusive electrons, (c) single vertex and $(d)$ double vertex techniques. Also shown are the JETSET 7.3 prediction as tuned using all DELPHI data and the expected background. 
I. The most energetic jet was taken as a quark jet, and cuts on $P_{J}$ were applied to each of the two lower energy jets in order to establish which was the quark and which was the gluon jet. The main criterion applied was to demand that one of the two lower energy jets satisfied the condition $P_{J}<0.01$. The remaining jet was then taken as the gluon provided its probability value, $P_{J}$, did not fall below 0.1 . This latter cut ensured that the decay products of the $b$ hadrons did not, in general, filter through to the selected sample of gluon jets. A total of 23138 (DURHAM) or 24643 (JADE) gluon jets were selected using this single vertex tag method.

II. Both quark jets were identified by applying cuts to the jet probability variable. By demanding that two of the three jets satisfied the condition $P_{J}<0.01$, the remaining jet was then considered as the gluon provided its probability value, $P_{J}$, exceeded 0.1 . Note that no energy requirement for the tagging of quark jets was applied, leading to the selection of a few events in which the gluon induced jet carried the largest fraction of energy. A total of 6382 (DURHAM) or 6791 (J ADE) gluon jets were selected using this double vertex tag method.

For the symmetric event topologies looser cuts could be used as the quark and gluon jet properties were obtained using a subtraction technique [21]. Only events with a signature of $b$ quark induced events were selected as input to the gluon identification by demanding that $P_{N}$, for the whole event, did not exceed a value of 0.032 . For Y events the procedure followed method I, however demanding $P_{J}>0.1$ for the gluon jets and $P_{J}<0.1$ for heavy quark jets. A number of 8238 gluon jets in $Y$ type events were selected using this cut. For Mercedes type events both of the quark jets had to be identified as all of the three jets had comparable energy (as in method II). $P_{J}<0.1$ was required for both of the quark jets and $P_{J}>0.1$ for the gluon candidates, respectively. In total, 568 gluon jets were identified within Mercedes events [21].

The present quark and gluon jet selection collects samples of jets whose energies are not restricted to a fixed value. The energy spectra of the gluon jets and expected background for each of the methods used are shown in figure 2. The purest gluon sample was obtained with the double vertex anti-tagging technique. It also contains gluon jets with energies above $35 \mathrm{GeV}$.

\section{Quark and Gluon Jet Purities}

The purities of the tagged gluon jet samples were evaluated using the JETSET 7.3 event generator [22], with full simulation of the DELPHI detector [23], by associating each reconstructed jet in the detector to an underlying quark or gluon jet. More specifically, the jet finding algorithm was applied to the final state partons at the end of the QCD shower and a value of $y_{c u t}$ was chosen such that three jets were always reconstructed. The two jets containing the primary quarks were labelled quark jets while the remaining jet was considered as the gluon jet. Each jet at the detector level was then associated to that quark or gluon jet at the parton level which best matched its direction. This minimised the probability of assigning hadrons with secondary vertices to gluon jets [20]. The gluon purity of each 'anti-tagged' gluon sample was then given by that fraction associated to the underlying gluon jet.

To avoid double counting of jets due to the different tagging methods, jets simultaneously tagged by two or more of these methods were assigned to the method which provided the highest purity. 
The gluon purities achieved for each of the above methods were studied as a function of $y_{c u t}$ and the gluon energy, for both DURHAM and JADE recombination schemes [20]. The double vertex tagging method gave the highest purity of up to $94 \%$ whereas the other methods gave $85 \%$ to $87 \%$. Another nice feature of the double vertex tagging method was the stability of the gluon purity down to $y_{\text {cut }}$ values lower than those reached by the other methods considered in the present analysis.

Purities of the quark jets in the $q \bar{q} \gamma$ event sample were also estimated using simulated data. On average, the quark purity for the events selected by the DURHAM algorithm was $92 \%$ whereas $94 \%$ purity was achieved when the JADE scheme was applied. There were weak dependencies of the purities on the energy and $y_{c u t}$. The flavour composition of the selected events was also studied and found to be consistent with the hypothesis that the photons were radiated by the final quarks according to their electromagnetic charge squared [20].

Due to the lower purities chosen for the symmetric events, gluon jet purities had to be evaluated very carefully for these special configurations. In order to reduce possible ambiguities in the assignment of partons to the jets, heavy hadrons were associated to the jets using the full detector simulation by two independent means [21]. On the one hand it was assumed that the jet which had the largest angle to the heavy hadrons would be the gluon induced jet (angle assignment), on the other hand the jet containing the fewest decay particles from the heavy hadrons was assigned to the gluon (history assignment).

\begin{tabular}{|c|c|ccc|}
\hline \multirow{2}{*}{ Method } & \multicolumn{3}{|c|}{ Angle assignment } \\
\cline { 2 - 5 } & gluon in: & Jet & Jet 2 & Jet 3 \\
\hline \multirow{3}{*}{ History } & Jet 1 & $5.3 \%$ & $0.05 \%$ & $0.09 \%$ \\
assignment & Jet 2 & $0.01 \%$ & $34 \%$ & $0.5 \%$ \\
\hline
\end{tabular}

Table 4: Correlation of angle and history assignment. The values in this table have been calculated for arbitrary three jet events with $\theta_{2,3}^{\text {proj }} \in\left[110^{\circ}, 170^{\circ}\right]$, with $\theta_{2,3}^{\text {proj }}$ being the angle between jets 2 and 3 in the event plane.

Table 4 shows that both methods are highly correlated and that therefore the purities can be estimated with small systematic uncertainties. By the identification described above gluon jet purities of $80.0 \% \pm 2.0 \%$ (Y events) and $73.2 \% \pm 2.5 \%$ (Mercedes events) were achieved [21].

\section{Results}

The tagging methods provided samples of quark and gluon jets for comparative studies of quark and gluon fragmentation as a function of the jet energy. In this section we discuss the charged particle multiplicity and the semi-inclusive distributions sensitive to the dynamics of quark and gluon jet fragmentation. 


\subsection{Charged Particle Multiplicities in Quark and Gluon Jets}

The charged particle multiplicity distribution $F_{n}^{i, \text { unf }}$ of the selected jets included in the non-symmetric $q \bar{q} g / q \bar{q} \gamma$ configurations was unfolded from the measured distribution $F_{m}^{i, o b s}$ by constructing an acceptance matrix $A_{m n}^{i}$ for each energy interval $i$ using the full detector simulation $\left(F_{m}^{i, o b s}=A_{m n} F_{n}^{i, u n f}\right)$. The elements of this acceptance matrix $A_{m n}^{i}$ denoted the probability of a jet with original multiplicity $n$, including charged particles from all $K^{\circ}$ and $\Lambda$ decays, to be observed as a jet with $m$ charged particles, accounting for the event and track selection efficiencies and for the additional spurious tracks arising from hadron interactions in the detector material and from photon conversions. In order to reduce the complexity of the correction procedure, the multiplicity distribution was approximated by a negative binomial distribution [27] (NBD) whose free parameters, namely the mean $(n)$, the dispersion $(k)$, and the normalisation $(N)$ were adjusted by a fitting method [20]. The applicability of the negative binomial distributions was extensively tested using simulated events for all energy points. In all instances the NBD was able to describe the mean of the true multiplicity to within $0.2 \%$, and by applying the full method, the original mean value of the true multiplicity could be reproduced within $1 \%$.

The mean multiplicity attributed to the gluon jet was extracted by simultaneously fitting all available data from the four selected samples at each energy point. The unfolded gluon multiplicity distribution was assumed to be composed of a mixture of the true gluon multiplicity, constant in all the four samples, and a background which depended on each particular sample. Only the purities were taken from the simulation whereas the parameters associated to the NBD were fitted according to:

$$
F_{n}^{i, \text { unf }}(s)=p^{i}(g, s) \cdot F_{n}^{i, \text { true }}(g)+\left(1-p^{i}(g, s)\right) \cdot F_{n}^{i, b a c k}(s),
$$

where $F_{n}^{i, u n f}(s)$ was the multiplicity distribution found in data, unfolded for detector effects, for each of the event samples: $s=\mu, e, 1 v t x, 2 v t x . F_{n}^{i, t r u e}(g)$ was the true charged multiplicity for gluon jets which was common to all the various sets of events and $F_{n}^{i, b a c k}(s)$ was the jet multiplicity of the background events for each of these sets. Finally $p(g, s)$ was the gluon purity as derived from simulation.

In a similar way, using the $q \bar{q} \gamma$ event sample, the mean value of the multiplicity distribution for quark jets was corrected applying the same technique although different sources of background were to be considered. In this case, the mean multiplicity per energy point was extracted according to:

$$
F_{n}^{i, \text { unf }}=p^{i}(q) \cdot F_{n}^{i, \text { true }}(q)+p^{i}(2 j e t s) \cdot F_{n}^{i, 2 j e t s}(2 j e t s)+p^{i}(\tau) \cdot F_{n}^{i, \tau}(\tau),
$$

where the considered contamination sources arises from two jet events with mis-identified pions $\left(F_{n}^{i, 2 j e t s}\right)$ and radiative $\tau^{+} \tau^{-}$events $\left(F_{n}^{i, \tau}\right)$. The values of $p(q), p(2 j e t s)$ and $p(\tau)$ were the fraction of events populating the photon sample according to the simulation.

The measured mean charged particle multiplicity as a function of the jet energy in both quark and gluon samples is shown in figure 3(a) for the DURHAM algorithm. The JETSET prediction is seen to be in reasonable good agreement with the data. In table 5 the parameters $n$ and $k$ for the fitted NBDs are also shown.

In figure $3(\mathrm{~b})$, the ratio, $r(E)$, of the mean charged particle multiplicities in the tagged gluon and quark jet samples is shown as a function of the jet energy. The value of this ratio varies from $1.06 \pm 0.18$ at $10 \mathrm{GeV}$ to $1.38 \pm 0.09$ at $40 \mathrm{GeV}$ suggesting already an energy dependence of the charged multiplicity ratio. A linear fit to the energy dependence using only data from the non-symmetric topologies yields 
$\Delta r / \Delta E=(105 \pm 34$ (stat.) \pm 18 (syst.) $) \cdot 10^{-4} \mathrm{GeV}^{-1}$. The average value of $r$ over the full energy range is $\langle r\rangle=1.232 \pm 0.026$ (stat.) \pm 0.018 (syst.).

The estimation of the systematic error includes the uncertainties due to the finite statistics in the simulation used to calculate the sample purities and the limitations inherent to the fitting/unfolding procedure described previously. The size of the uncertainties arising from a possible non-perfect modelling of all the various simulated background jets entering in the gluonic sample were quantified as follows. For each energy interval, the gluon jet purity was changed by re-scaling the quark jet background to account for the exact differences between the measured and the simulated gluon jet energy distributions as shown in figure 2 . The new mean of the charged multiplicity distribution was then obtained using these new gluon purity factors and the differences between the old and the new mean values were calculated for each energy point. The differences found are rather small as compared to the statistical error reaching values from $\sim 0.5 \%$ at $10 \mathrm{GeV}$ gluon energy to $\sim 3 \%$ at $40 \mathrm{GeV}$ gluon energy. The effects due to the poor statistics present in some energy intervals of the quark jet sample were also investigated. For this purpose, the mean charged multiplicity of the quark jets was fitted using different bin sizes when describing the distribution. The change in the fitted results is negligible for data points with energies above $25 \mathrm{GeV}$ but it has some influence in the mean values below this energy, always, however, within statistical errors. This effect is in fact the larger contribution entering the quoted systematic error of the slope measurement. The mean quark multiplicities values and their associated errors shown in figure 3(a) and in table 5 correspond to the results obtained when grouping the multiplicity distributions in bins of two particles.

The average corrections to the absolute measured mean charged quark and gluon jet multiplicities have been found to lie in the ranges $(12 \pm 4) \%$ and $(14 \pm 6) \%$, respectively. For $r$ the corresponding average correction factor is $\sim 4 \%$ as both quark and gluon correction factors usually compensate each other. Only the $r$ value of the last energy point at $40 \mathrm{GeV}$ had to be corrected by a larger factor of $\sim 10 \%$, since at this point, a larger gluon background is present in the sample.

\begin{tabular}{|c||r|r||c|r|}
\hline Energy [GeV] & \multicolumn{1}{|c|}{$n_{\text {gluon }}$} & \multicolumn{1}{|c|}{$k_{\text {gluon }}$} & \multicolumn{1}{c|}{$n_{\text {quark }}$} & \multicolumn{1}{|c|}{$k_{\text {quark }}$} \\
\hline \hline 10 & $5.78 \pm 0.06$ & $24.1 \pm 6.1$ & $5.43 \pm 0.90$ & $7.3 \pm 5.2$ \\
15 & $6.64 \pm 0.09$ & $15.0 \pm 1.7$ & $5.54 \pm 0.43$ & $6.0 \pm 2.5$ \\
20 & $8.18 \pm 0.17$ & $9.6 \pm 1.4$ & $7.52 \pm 0.36$ & $13.3 \pm 9.1$ \\
25 & $9.13 \pm 0.14$ & $8.9 \pm 1.0$ & $7.38 \pm 0.33$ & $11.8 \pm 5.4$ \\
30 & $9.83 \pm 0.30$ & $6.0 \pm 0.7$ & $7.89 \pm 0.35$ & $21.3 \pm 9.9$ \\
35 & $10.67 \pm 0.33$ & $12.4 \pm 6.9$ & $8.24 \pm 0.17$ & $17.6 \pm 5.6$ \\
40 & $11.86 \pm 0.68$ & $3.8 \pm 2.3$ & $8.61 \pm 0.20$ & $14.9 \pm 1.4$ \\
\hline
\end{tabular}

Table 5: Values of the observed mean multiplicities $(n)$ and dispersions $(k)$ for the quark and gluon jet samples as a function of the jet energy. These values are derived from the fit to Negative Binomial Distributions using the DURHAM algorithm at $y_{c u t}=0.01$ for the jet reconstruction.

Using $Y$ and Mercedes event configurations, similar information could be obtained from the analysis of tagged gluon jets and the mixed sample. The charged multiplicities in the tagged and in the mixed sample ( $n_{t a g}$ and $n_{m i x}$, respectively) followed from the 

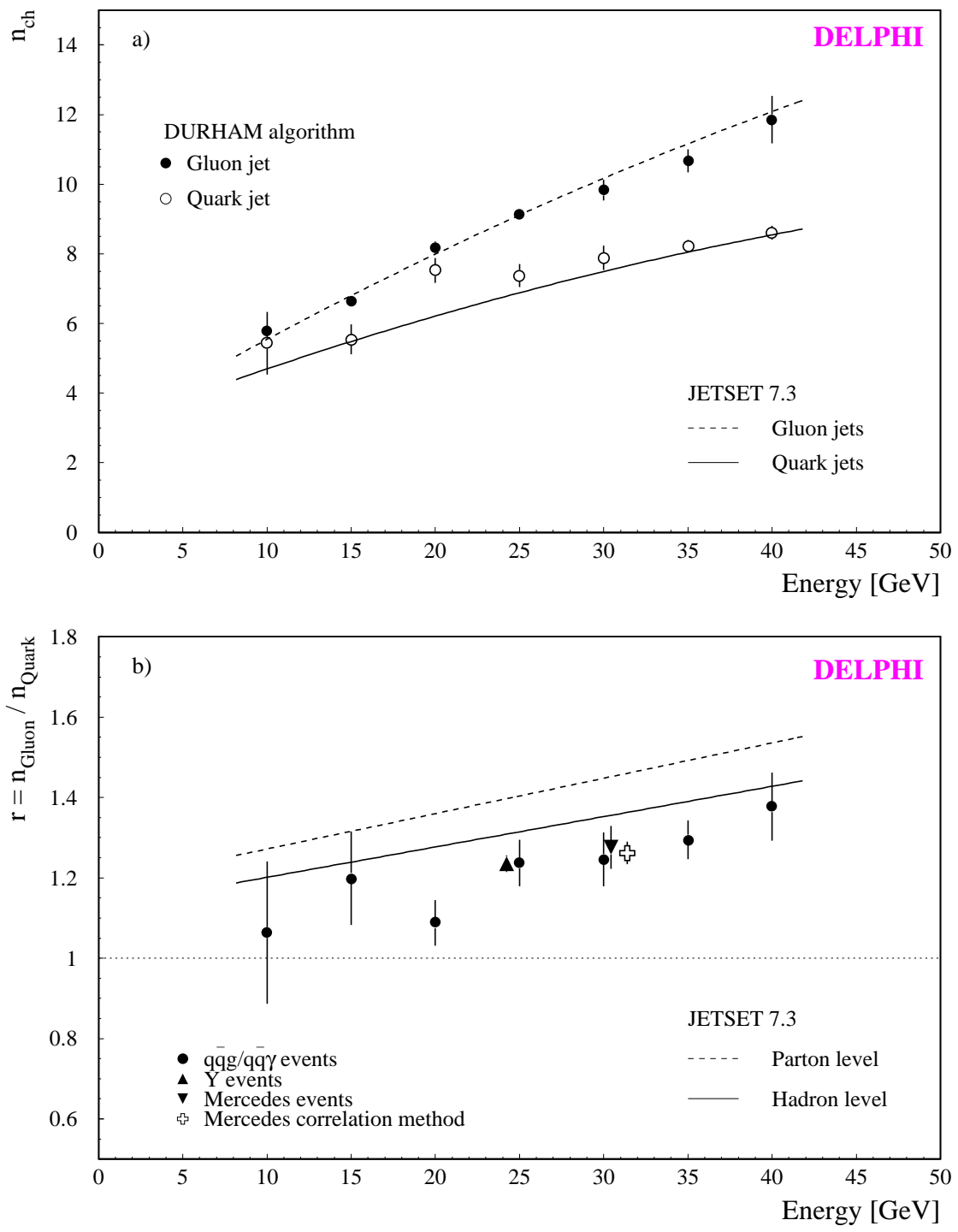

Figure 3: (a) The mean charged particle multiplicities for quark and gluon jets and (b) their ratio $r$ as a function of the jet energy. The JETSET 7.3 curves represent the model prediction as tuned using all DELPHI data. Also, notice that the values shown here correspond to the corrected values, in the case of symmetric events, for having the same $b$ and $c$ quark content as that obtained in the tagged $q \bar{q} g / q \bar{q} \gamma$ sample, as it is explained in the text. The data point of the correlation method in (b) is shifted by $+1 \mathrm{GeV}$ for better display. 
equations:

$$
\begin{aligned}
& n_{\text {tag }}=p(g)_{\text {tag }} \cdot n(g)+\left(1-p(g)_{\text {tag }}\right) \cdot n(q) \\
& n_{\text {mix }}=p(g)_{\text {mix }} \cdot n(g)+\left(1-p(g)_{\text {mix }}\right) \cdot n(q),
\end{aligned}
$$

where $n(q)$ and $n(g)$ denoted the true underlying mean charged multiplicities in the quark and gluon jets, respectively; $p(g)_{\operatorname{tag}}$ and $p(g)_{\text {mix }}$ were the purities of gluons in the tagged and the mixed sample. In the mixed sample only events which had failed the $b$ tag at event level were used. This kept influences of $b$ decays small. The purities were taken from event simulation and were the only model dependent assumption entering in the analysis. Eq. 2 could be solved to yield the measured multiplicities for quark and gluon jets.

For the ratio of the charged multiplicities in quark and gluon jets, an acceptance correction was made directly to the mean value of the multiplicity distribution. The ratio of the multiplicities for quark and gluon jets is obtained to be:

$$
\begin{aligned}
& \left.\left.r\left(E_{Y}\right)=1.279 \pm 0.021 \text { (stat. }\right) \pm 0.020 \text { (syst. }\right) \\
& \left.r\left(E_{\text {Mercedes }}\right)=1.323 \pm 0.053(\text { stat. }) \pm 0.020 \text { (syst. }\right) .
\end{aligned}
$$

The systematic error is dominated by the uncertainty of the gluon jet purity in the tagged sample.

A further measurement of the ratio $r$ was obtained by applying a novel correlation method to the untagged Mercedes events [28]. The analysed data sample included the whole period 1991-1994, hence the intrinsic statistical error was smaller than those obtained in the other methods. This correlation function $C\left(n_{1}, n_{2}, n_{3}\right)$ was defined as follows:

$$
C\left(n_{1}, n_{2}, n_{3}\right)=\frac{P\left(n_{1}, n_{2}, n_{3}\right)}{P_{\text {uncor }}\left(n_{1}, n_{2}, n_{3}\right)} .
$$

Here $P\left(n_{1}, n_{2}, n_{3}\right)$ was the probability to observe an event with charged particle multiplicities in the three jets equal to $n_{1}, n_{2}$, and $n_{3}$, respectively $\left(n_{1} \geq n_{2} \geq n_{3}\right)$. The corresponding uncorrelated probability $P_{\text {uncor }}\left(n_{1}, n_{2}, n_{3}\right)$ was constructed by using a jet mixing method which consisted of constructing fake events from three subsequent real events whose original jets were randomly taken and associated to form an artificial three jet event. The uncorrelated probability $P_{\text {uncor }}$ could then be expressed by the multiplicity distributions of gluon and quark jets which were assumed to have about the same energy, $\sim 30.4 \mathrm{GeV}$, and to be described by NBDs whose parameters were correlated by the $r\left(E_{\text {Mercedes }}\right)_{\text {correlation }}=n_{\text {gluon }} / n_{\text {quark }}$ parameter with the constraint $n_{\text {gluon }}+2 \cdot n_{\text {quark }}=n_{\text {event }}$. A fit to the measured correlation function $C$ yields:

$$
\left.r\left(E_{\text {Mercedes }}\right)_{\text {correlation }}=1.253 \pm 0.028 \text { (stat. }\right) \pm 0.044 \text { (syst.) }
$$

if the DURHAm algorithm with $y_{c u t}=0.015$ is used to select Mercedes type three jet events with angles of $120^{\circ} \pm 20^{\circ}$ between the jets in the event plane.

The various methods used in the present analysis are based on different sources of quark jet samples which contain different quark flavour compositions. As a consequence of this, the individual measured multiplicities need to be corrected to enable a proper comparison and combination of the results. The symmetric configuration analyses were thus corrected to account for the same $b$ and $c$ quark content as the one present in the tagged $q \bar{q} \gamma$ sample which was about $11 \%$ and $33 \%$, respectively, and was chosen as the reference sample. The correction has been performed on the basis of the charged multiplicity difference reported in references [29] and [30] whose average values have been estimated as $2.96 \pm 0.33$ (stat. + syst.) for $b \bar{b}$ events and as $1.14 \pm 0.62$ (stat. + syst.) 
for $c \bar{c}$ events. As it is also discussed in these works no energy dependence is assumed for these differences. In the case of tagged $\mathrm{Y}$ and Mercedes events the $b$ correction, $2.2 \%$, decreases the value of the observed $r$ because this analysis explicitly excludes $b$ quark jets. For the correlation method, on the contrary, the value of $r$ is increased because the normal fraction of $b$ quarks at the $Z$ peak of $\sim 22 \%$ is considered. The correction accounting for the $c$ multiplicity is common to both analyses which assume the standard composition defined by the coupling to the $Z$. It lowers down the values of $r$ by an additional $1.1 \%$. Table 6 and figure 3 summarise all $r$ values measured by the various analyses once these corrections are applied.

\begin{tabular}{|l|c|c|}
\hline Method/configuration & Energy $[\mathrm{GeV}]$ & $r$ \\
\hline \hline Tagged Y events & $24.2 \pm 0.02$ & $1.235 \pm 0.021 \pm 0.022$ \\
Tagged $q \bar{q} g / q \bar{q} \gamma$ & $26.6 \pm 0.70$ & $1.232 \pm 0.022 \pm 0.018$ \\
Tagged Mercedes events & $30.4 \pm 0.03$ & $1.276 \pm 0.055 \pm 0.022$ \\
Mercedes events, correlation method & $30.4 \pm 0.02$ & $1.263 \pm 0.029 \pm 0.044$ \\
\hline
\end{tabular}

Table 6: Values of $\left(n_{\text {gluon }} / n_{\text {quark }}\right)$. The values for symmetric events are corrected to account for the same $b$ and $c$ quark content as that obtained in the tagged $q \bar{q} g / q \bar{q} \gamma$ sample.

The data used in the different analyses partially overlap. To remove any correlation only the input associated to the smallest error per data point has been entered when calculating an average multiplicity ratio for $r$. The result is:

$$
<r>=1.241 \pm 0.015 \text { (stat.) } \pm 0.025 \text { (syst.) }
$$

The enhanced charged multiplicity in gluon jets w.r.t. quark jets is therefore proved and its average value is found to be in reasonable agreement with previous observations obtained by other experiments $[2,3]$ once the proper corrections to account for the same composition of $b$ and $c$ quark jets in the quark sample are considered.

The value of $r$ can be further corrected to only account for the multiplicity of the light quarks: $u, d, s$. In this case it is:

$$
<r_{u d s}>=1.305 \pm 0.016 \text { (stat.) } \pm 0.032 \text { (syst.) }
$$

where the uncertainties of the $b$ and $c$ charged multiplicity measurements are considered in quadrature and are included in the systematic error.

The most novel experimental result of the present analysis is the increase of this ratio with the jet energy. A fit presupposing a linearly increasing ratio yields for the slope of $r(E)$ :

$$
\Delta r / \Delta E=(86 \pm 29 \text { (stat.) } \pm 14 \text { (syst.) }) \cdot 10^{-4} \mathrm{GeV}^{-1},
$$

where the quoted systematic error mainly accounts for the spread in the fit results when grouping the quark multiplicity distributions of the $q \bar{q} \gamma$ sample in bins containing one or two particles. The significance of the energy dependence of $r$ is $2.7 \sigma$. The result for the $\chi^{2} / n$.d.f. of this fit is 0.8 . A $\chi^{2} / n$.d.f. of 2.2 is instead obtained for the hypothesis of no energy dependence with the value of $r$ fixed at the average value measured above.

This behaviour is also found to be consistent with the JETSET prediction at both parton and fragmentation level [31]. The slopes obtained for the QCD model curves are $\Delta r / \Delta E=(90 \pm 3) \cdot 10^{-4} \mathrm{GeV}^{-1}$ at parton level and $\Delta r / \Delta E=(76 \pm 2) \cdot 10^{-4} \mathrm{GeV}^{-1}$ after 
fragmentation. Differences in the slopes and offset values are attributed to fragmentation effects. Their relative importance however decreases with increasing energy. The choice of the mass scale parameter $Q_{0}$ at which the parton shower evolution stops and the fragmentation takes over affects the parton level prediction. By varying the value of $Q_{0}$ in the range from $1 \mathrm{GeV}$ to $3 \mathrm{GeV}$, the prediction of the $r$ offset at parton level changes by $20 \%$ while the overall variation in the slope is kept within $5 \%$. The studied systematic uncertainties in the slope are thus well below the statistical error and therefore the slope measurement is, at present, largely dominated by the statistical error of $\sim 30 \%$.

\begin{tabular}{|c||r|r||c|c|}
\hline Energy [GeV] & \multicolumn{1}{|c|}{$n_{\text {gluon }}$} & \multicolumn{1}{|c|}{$k_{\text {gluon }}$} & $n_{\text {quark }}$ & \multicolumn{1}{|c|}{$k_{\text {quark }}$} \\
\hline \hline 10 & $7.04 \pm 0.10$ & $9.8 \pm 1.2$ & $5.44 \pm 0.85$ & $15.3 \pm 6.9$ \\
15 & $7.95 \pm 0.14$ & $9.3 \pm 1.1$ & $6.73 \pm 0.81$ & $15.1 \pm 3.5$ \\
20 & $9.35 \pm 0.19$ & $6.2 \pm 1.0$ & $7.46 \pm 0.53$ & $23.0 \pm 12.6$ \\
25 & $10.16 \pm 0.43$ & $6.5 \pm 1.4$ & $7.50 \pm 0.33$ & $12.8 \pm 8.4$ \\
30 & $11.18 \pm 0.47$ & $3.7 \pm 0.9$ & $8.19 \pm 0.19$ & $10.2 \pm 2.8$ \\
35 & $11.27 \pm 0.74$ & $6.6 \pm 1.2$ & $8.20 \pm 0.23$ & $9.1 \pm 1.7$ \\
40 & $12.61 \pm 1.32$ & $12.2 \pm 10.5$ & $8.41 \pm 0.16$ & $16.3 \pm 1.5$ \\
\hline
\end{tabular}

Table 7: Values of the observed mean multiplicities $(n)$ and dispersions $(k)$ for the quark and gluon jet samples as a function of the jet energy. These values are derived from the fit to Negative Binomial Distributions using the JADE algorithm at $y_{c u t}=0.04$ for the jet reconstruction.

When the same analysis was performed for the JADE scheme, with the quark jet mixture as defined by their coupling to photons, the average value $\langle r\rangle=1.369 \pm$ 0.019 (stat.) \pm 0.035 (syst.) was obtained from the tagged $q \bar{q} g / q \bar{q} \gamma$-analysis and the correlation method. As can be observed when comparing table 5 and table 7 , the mean charged multiplicities of the gluon sample are systematically greater for JADE than the corresponding ones for DURHAM whereas for the quark sample they are similar. The measured slope is $\Delta r / \Delta E=(87 \pm 49) \cdot 10^{-4} \mathrm{GeV}^{-1}$ as compared to a predicted value of $\Delta r / \Delta E=(120 \pm 5) \cdot 10^{-4} \mathrm{GeV}^{-1}$ at parton level. The significance of this result is now $1.8 \sigma$ mainly due to the lower statistics entering this analysis, as only data from the tagged $q \bar{q} g / q \bar{q} \gamma$ and Mercedes events in the correlation method, at $y_{c u t}=0.06$, have been used. The obtained value of $r$ in the JADE scheme is higher than the one for the DURHAM algorithm. This result could be expected from the studies performed in [19], the reason being a consequence of the property of the JADE algorithm which associates to each jet more soft particles at large angles than DURHAM does. Whether this increase is interpreted as being more sensitive to the QCD behaviour or just a feature of the algorithm is a delicate question to be answered. It can be deduced that only the different angular coverage of each of these two algorithms for a given fixed jet energy $[19,20]$ can account for the different results for $r$. This interpretation is also supported by a recent publication from OPAL [2] in which it is demonstrated that $r$ is hardly influenced by the value of $y_{\text {cut }}$ used to reconstruct jets. Therefore, the observed difference in the present study cannot be attributed to a non-optimal selection of $y_{c u t}$. Independent of the absolute values obtained for $r$ the increasing behaviour with energy is proved to be present at $2.7 \sigma$ significance level for DURHAM and at $1.8 \sigma$ significance level for JADE. Agreement with the parton shower prediction for the slope of $r$ is obtained in both cases. 
These results thus indicate that $r$ depends on the jet energy and also on the reconstruction jet algorithm used, mainly because of the intrinsic angular acceptance of the algorithm. Fragmentation effects, as shown in figure 3, decrease the value of $r$ w.r.t. what the QCD parton shower approximation predicts as implemented in JETSET and tuned by DELPHI. Still the measured value of $r$ is systematically smaller than that predicted by JETSET throughout the whole energy range. The increasing trend of $r$ as a function of the jet energy is however seen to follow the QCD-like expectation, at both parton level and after fragmentation.

\subsection{Topological Variables}

Sizeable differences are expected between distributions of hadrons in quark and gluon jets sensitive to the dynamics of quark and gluon fragmentation. To explore these differences and their possible energy dependence we study distributions as a function of

- the scaled energy

$$
x_{E}=\frac{E_{\text {part }}}{E_{\text {jet }}^{\text {calc }}},
$$

- the rapidity of the leading particle

$$
\eta=\frac{1}{2} \ln \left(\frac{E+p_{L}}{E-p_{L}}\right),
$$

- and the jet broadness

$$
\beta=\ln \left(\frac{3}{2} \frac{\sum p_{T}^{2}}{\sum p^{2}}\right),
$$

here $E_{\text {part }}$ is the particle energy while $p_{L}$ and $p_{T}$ are the parallel and transverse particle momentum w.r.t. the jet direction.

The distributions discussed in this sections are based on the DURHAM jet definition and are fully corrected for limited detector acceptance and resolution. The $\pi$ mass is assumed for all particles.

Figure 4 compares the scaled energy distribution for quark and gluon jets as obtained from $\mathrm{Y}$ and Mercedes events. As expected both selections lead to the same general pattern. The observed decrease is however more pronounced in gluon than in quark jets. Only at small $x_{E}\left(x_{E} \leq 0.2\right)$ where most of the particles are observed, the multiplicity in gluon jets is larger than in quark jets. The relative difference of the energy distributions here is $25-50 \%$ consistent with the the observed difference in total multiplicity. In the high momentum region the multiplicity in gluon jets is suppressed (by about one order of magnitude) w.r.t. quark jets. This is interpreted as due to the presence of the initial quark as a a valence quark inside one of the produced hadrons, whereas in case of an initial gluon emission all quarks have to be created in the fragmentation process.

Both the quark distributions of $\mathrm{Y}$ and Mercedes events are in excellent agreement with the overall scaled energy distributions from experiments at lower energies. For comparison the $x_{E}$ distributions of AMY [32] and TASSO [33] have been normalised to the number of jets, assuming it to be equal to two in general. Therefore particles originating from gluons are added to the two quark jets such that the overall $x_{E}$ distribution is expected to be found between the quark and the gluon distribution. Figure 4 shows that this is indeed the case for both $\mathrm{Y}$ and Mercedes type events. The distributions of quark jets and gluon jets cross at $x_{E} \sim 0.2$. 


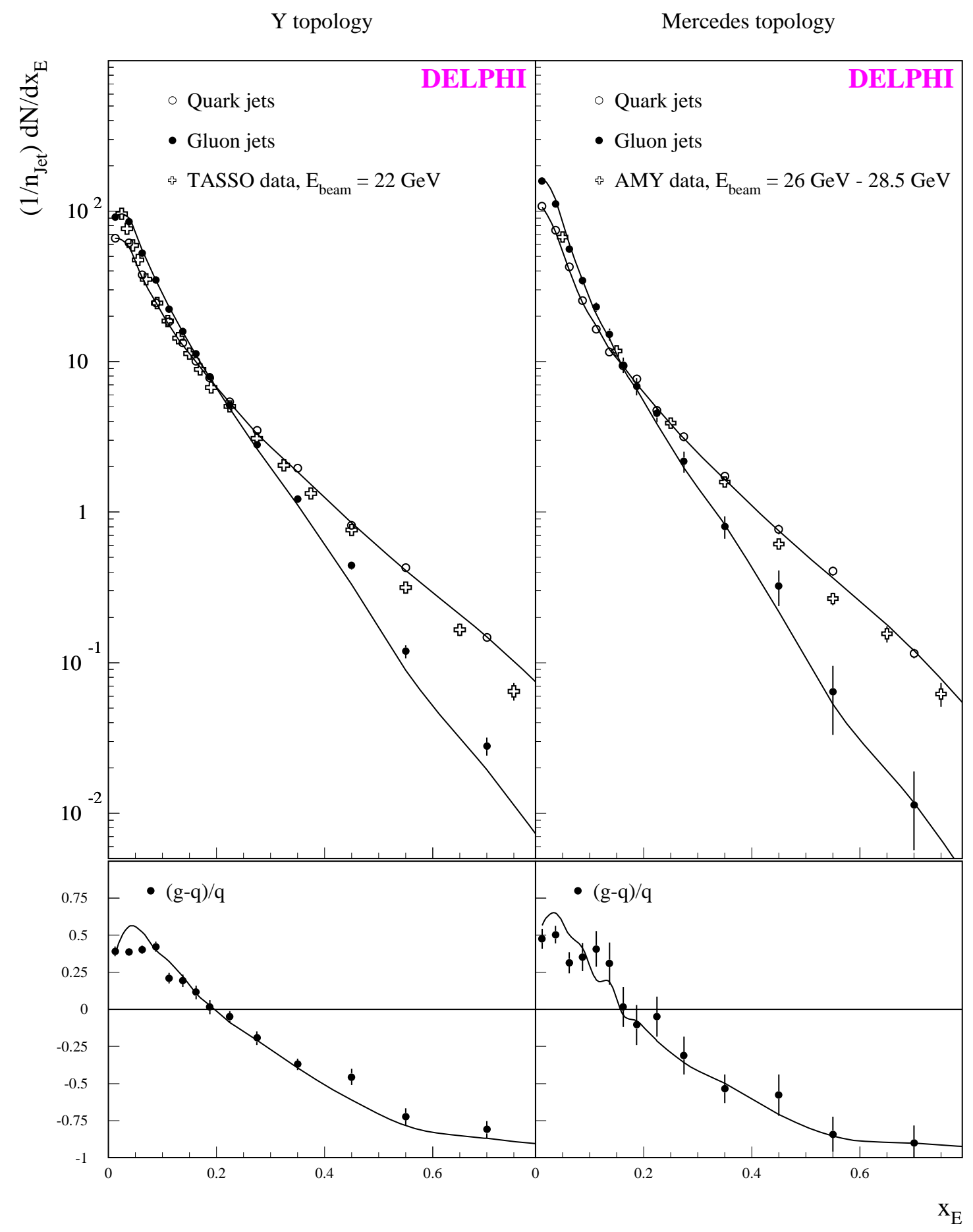

Figure 4: Scaled energy distributions for $\mathrm{Y}$ and Mercedes type events, the curves are the corresponding JETSET predictions. 


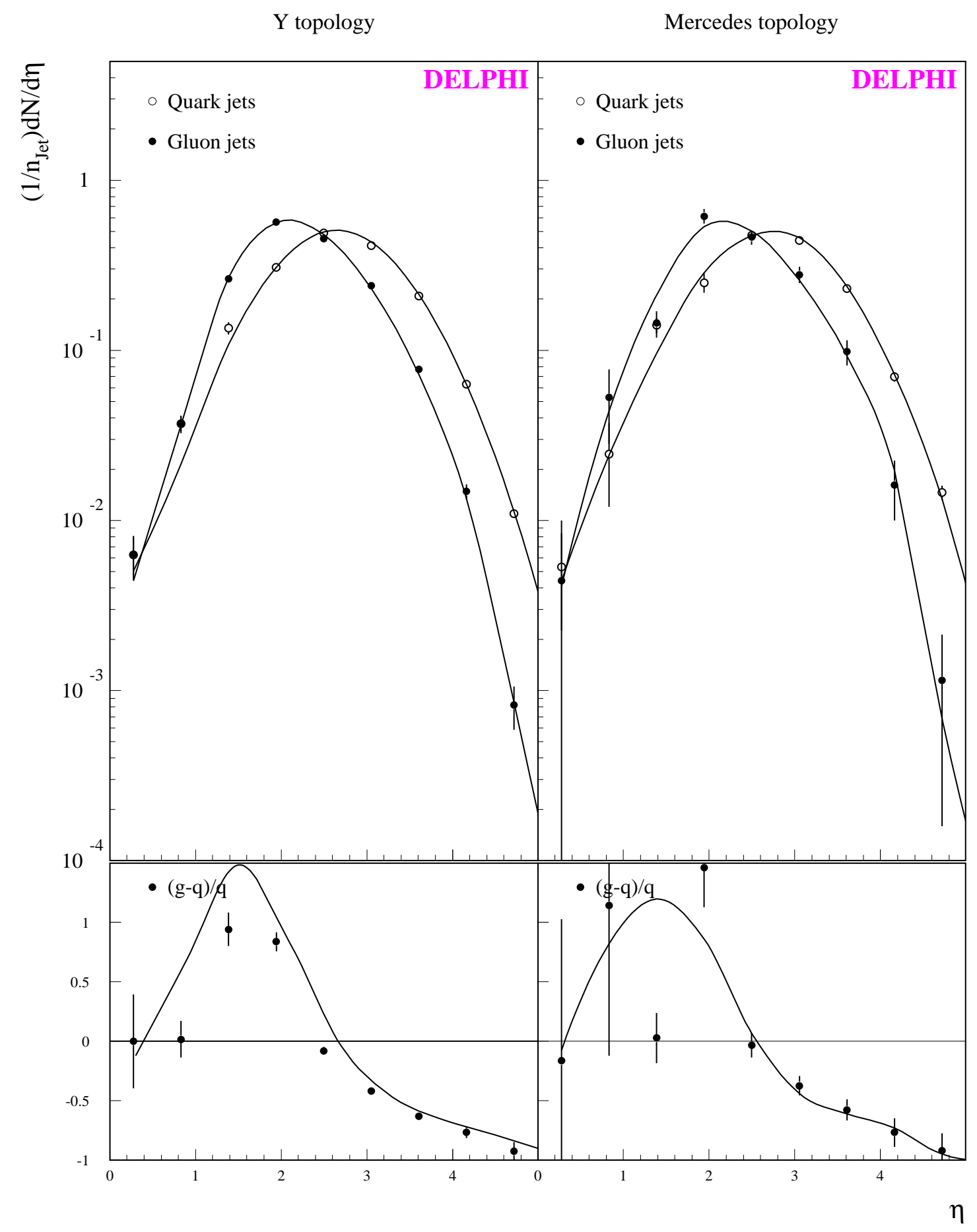

Figure 5: Rapidity distributions for $\mathrm{Y}$ and Mercedes type events, the curves are the corresponding JETSET predictions. 


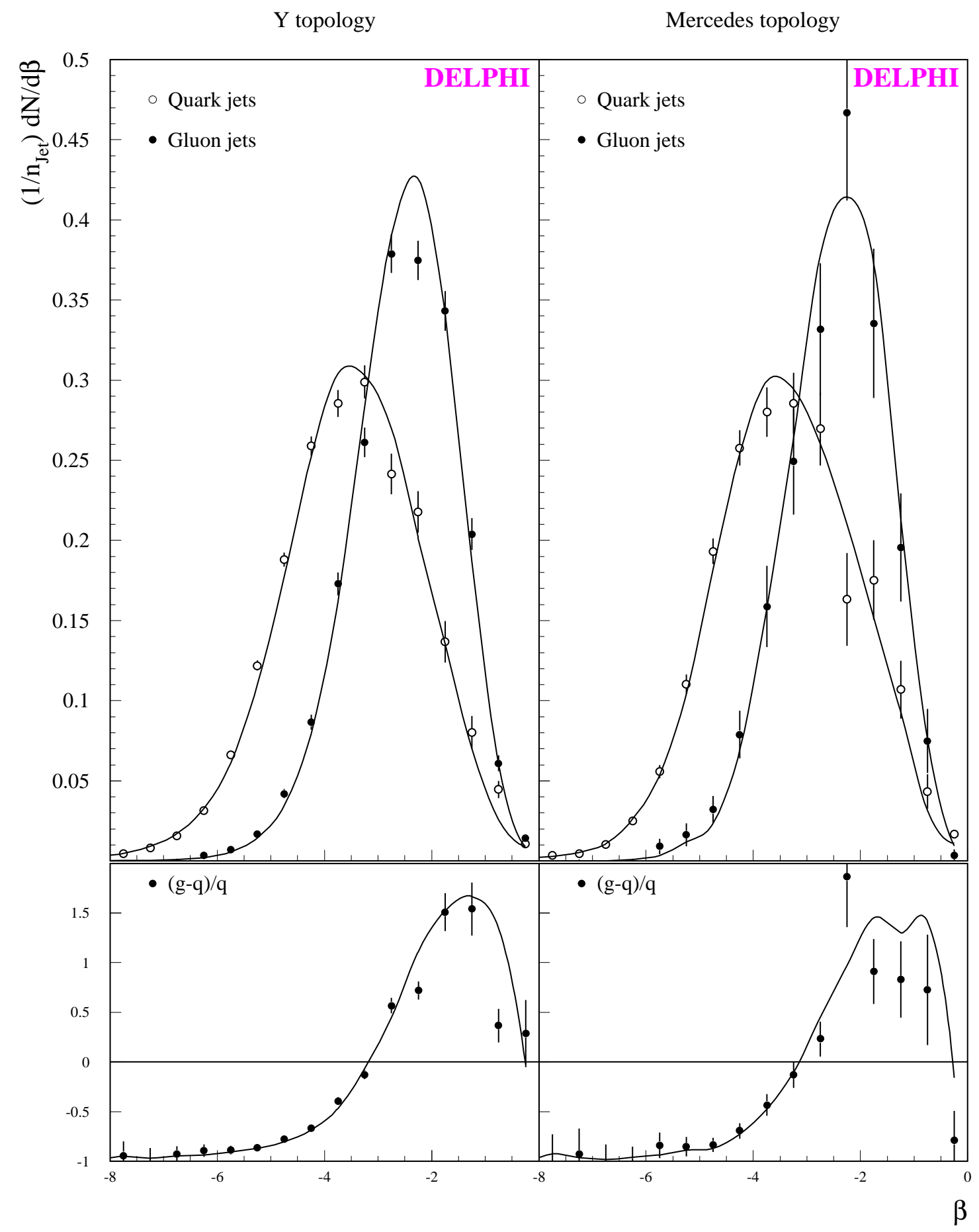

Figure 6: Jet broadness distributions for $\mathrm{Y}$ and Mercedes type events, the curves are the corresponding JETSET predictions. 

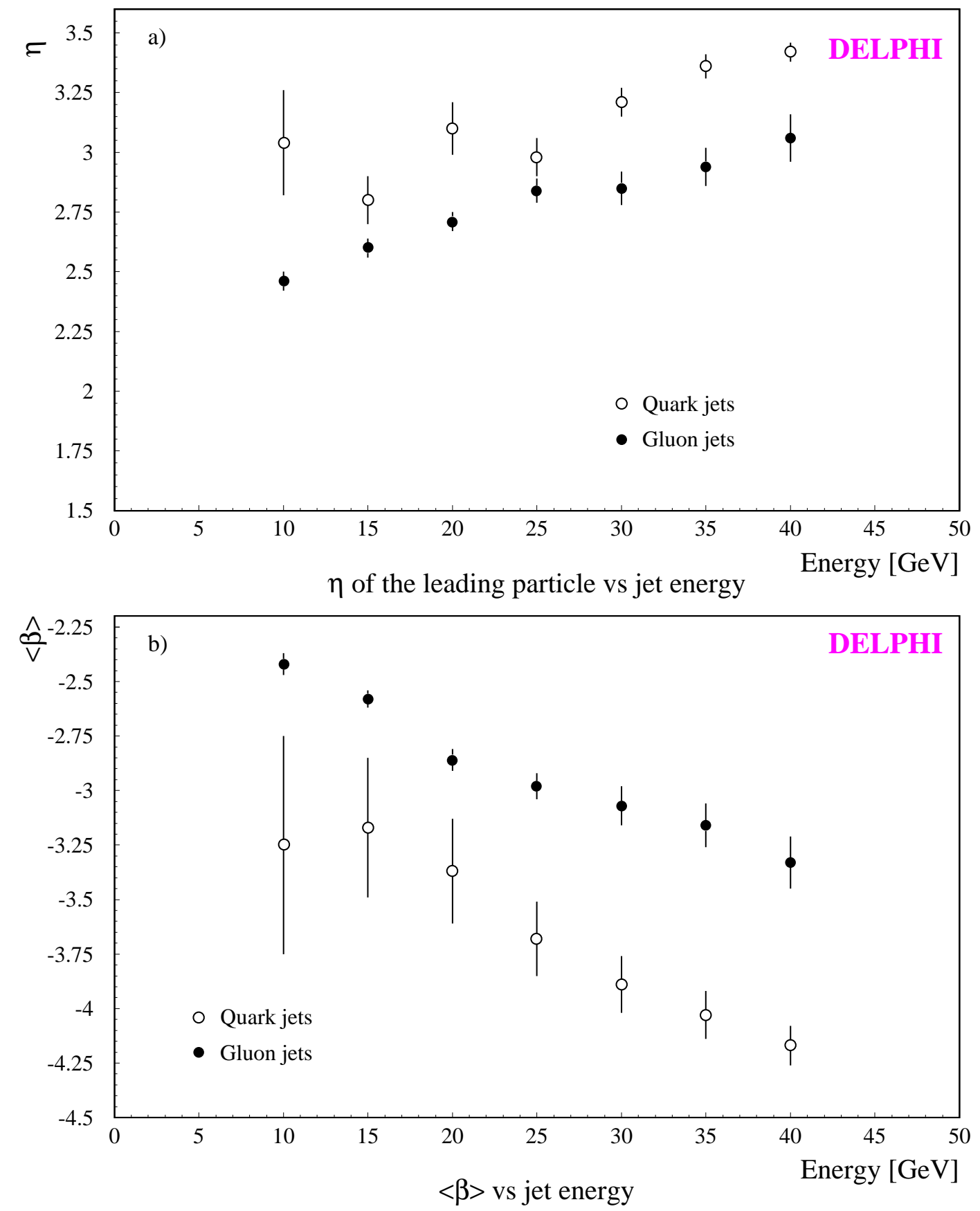

Figure 7: $\eta$ of the leading particle and $\langle\beta\rangle$ distributions as a function of the jet energies. 
In order to search for a possible energy dependence we compare integrals of the scaled energy distributions from quark and gluon jets for $\mathrm{Y}(\langle E\rangle=24.2 \mathrm{GeV})$ and Mercedes events $(\langle E\rangle=30.4 \mathrm{GeV})$. In the range $0.25 \leq x_{E} \leq 0.8$ the average multiplicity is decreased by the amount of $Q=-\left(14 \% \pm 3 \%\right.$ (stat) ) for quark jets. The $x_{E}$ region was chosen to be above $x_{E} \sim 0.2$ and include as high $x_{E}$ values as possible with sufficient statistics. The observed decrease is similar in magnitude to the one observed for charged hadrons in the same $x_{E}$ range from $e^{+} e^{-}$annihilation at lower energies (compare [34]). The same change in gluon initiated jets is $-(33 \% \pm 7 \%$ (stat)), thus a factor $2.4 \pm 0.5$ (stat) larger than for quark jets. Accepting the scaling violation process as the reason for the observed energy dependence a stronger energy dependence is indeed expected for gluon jets due to the higher colour charge of the gluon and thus the increased probability to radiate further gluons. Naively the ratio of the energy dependences for gluon and quark jets is related to the quark and gluon colour factors $Q=\left(C_{A}+\kappa \cdot n_{f} T_{f}\right) / C_{F} \simeq 2.5$. Here $n_{f}$ is the effective number of active quark flavours and $\kappa(\mathcal{O}(0.1))$ is an extra suppression factor expected due to different dynamics of gluon radiation and $g \rightarrow q \bar{q}$ splitting. Thus the observed energy dependence of the energy distributions may be taken as a qualitative indication that at large momenta the quark and gluon splitting processes take place as expected from QCD. However further studies and especially more data are needed to firmly establish this interpretation.

The longitudinal and transverse properties of jets can be addressed by the rapidity and the jet-broadness distributions. Figure 5 shows the comparison of the distribution of the rapidity corresponding to the most energetic particle in quark and gluon jets as determined again from $\mathrm{Y}$ and Mercedes events. The rapidity in quark jets reaches larger values than for gluon jets. Again this is understood because in case of the quark jet a leading hadron may incorporate the quark as a valence quark whereas in the gluon case quarks have to be created first by gluon splitting. The average leading hadron rapidity as function of energy is shown in figure 7(a). For all energies the average rapidity is about $\Delta \eta \simeq 0.5$ larger in the quark than in the gluon jets. For both types of jets the expected increase with energy is observed.

The $\beta$-variable defined in Eq. 3 is constructed to give a quantitative measure of the broadness of jets. The examination of $\mathrm{Y}$ and Mercedes events (figure 6 ) shows that gluon jets are wider than quark jets as expected from the different quark and gluon colour structure. The mean value of this variable for both quark and gluon jets is shown in figure $7(\mathrm{~b})$ as a function of jet energy. It is evident that although both quark and gluon jets become narrower with increasing energy, the gluon jet remains broader than its quark counterpart of equivalent energy.

\subsection{The String Effect}

Coherence phenomena are basic to any gauge theory. In QCD jet dynamics two classes of coherence occur: intra-jet and inter-jet coherence. The so called string effect corresponds to the latter class and deals with the angular structure of soft particle flow when three or more energetic partons are involved in the process. According to QCD in leading order, the particle angular distributions are predicted to depend on the geometry and colour topology of the whole jet ensemble [5] and, hence, measurable differences in the particle flow distributions are expected for:

- different inter-jet regions of the same initial $q \bar{q} g$ configuration, 
- same inter-jet region and same initial three jet event configuration but different colour nature, i.e., comparing $q \bar{q} g$ w.r.t. $q \bar{q} \gamma$.

The investigation of all these situations is discussed below.

So far the string effect has mainly been analysed using asymmetric events where angular regions of increased gluon density could be selected using jet energy ordering. In this analysis we investigate the string effect in symmetric $q \bar{q} g$ events where the quark jets are tagged using the double vertex method. Figure $8(\mathrm{a})$ presents the normalised differential particle flow as a function of the angle $\Psi$ of the particles w.r.t. the direction of the most energetic quark jet (oriented to the $2^{\text {nd }}$ quark jet) as determined from 568 Mercedes events. As expected the particle flow in the inter-jet region between the two quarks, opposite to the gluon is suppressed w.r.t. the inter-jet regions flanked by a quark and the gluon. This suggests that the string effect is also present in fully symmetric events and it is not an artifact of kinematic selections. Quantitatively comparing the minima located at $\pm\left[50^{\circ}, 70^{\circ}\right]$, the particle flow in the $q \bar{q}$ and in the $q g$ regions gives a ratio of the population asymmetry of $R_{g}=N_{q g} / N_{q \bar{q}}=2.23 \pm 0.37$ for the DuRHAM Jet definition.

Starting from the $q \bar{q} g / q \bar{q} \gamma$ event sample, $q \bar{q} g$ events satisfying the double vertex tag were selected with the additional constraint of fulfilling a Y event configuration in which the separation between the most energetic jet and the other two jets was required to be $\Delta \Theta=150^{\circ} \pm 10^{\circ}$. The $q \bar{q} g$ sample was thus composed by 558 events and its charged particle density is shown in figure 8(b) analogously to figure 8(a). The ratio of the charged particle flow has also been calculated for this case between the angular intervals $\pm\left[-35^{\circ},-115^{\circ}\right]$, leading to $R_{g}=N_{q g} / N_{q \bar{q}}=1.60 \pm 0.10$ with DURHAM and $R_{g}=N_{q g} / N_{q \bar{q}}=1.61 \pm 0.10$ with J ADE.

For further quantitative analysis the above set of $q \bar{q} g \mathrm{Y}$ events has been compared to the corresponding set of $84 q \bar{q} \gamma$ events with the same configuration. A cleaner verification of the string effect is then evident when comparing the particle flow in the $q \bar{q}$ region $\left[+35^{\circ},+115^{\circ}\right]$, with the corresponding region in $q \bar{q} \gamma$ events (figure $8(\mathrm{c})$ ). A value for the ratio of these particle densities without detector correction is measured to be:

$$
\left.\left.R_{\gamma}(2 v t x)=\frac{N_{q \bar{q}}(q \bar{q} g)}{N_{q \bar{q}}(q \bar{q} \gamma)}=0.56 \pm 0.06 \text { (stat. }\right) \pm 0.02(\text { acc. }+ \text { pur. }) \pm 0.01 \text { (jet alg. }\right)
$$

where the second error considers the effect of a possible correction due to the detector acceptance and to the gluon/quark jet purities of both event samples. It should be noticed that individual corrections of about $10 \%$ are to be applied for both the numerator and the denominator. However, they account for similar detector effects and most of them compensate in the ratio $R_{\gamma}(2 v t x)$. Selecting mainly $b \bar{b} g$ events by using the double vertex technique was not found to significantly affect this ratio at large angles of $75^{\circ} \pm 40^{\circ}$. The third quoted error corresponds to the dispersion on the result as given by the two jet finding schemes used in the analysis.

The reconstructed energies of the quark jets were summed up for each event and the average values of the $q \bar{q} \gamma$ and $q \bar{q} g$ data samples, about $60 \mathrm{GeV}$ for this configuration, were then computed. The corresponding value of the $q \bar{q} \gamma$ distribution was found to exceed that of the $q \bar{q} g$ distribution by $\sim 3 \%$. This is commonly understood as a kinematic factor originating in the artificial mass acquired after fragmentation by the gluon jet which decreases the energies of the quark jets. This energy shift however cannot account for the large difference in the particle flow observed in data which amounts to about $40 \%$. 

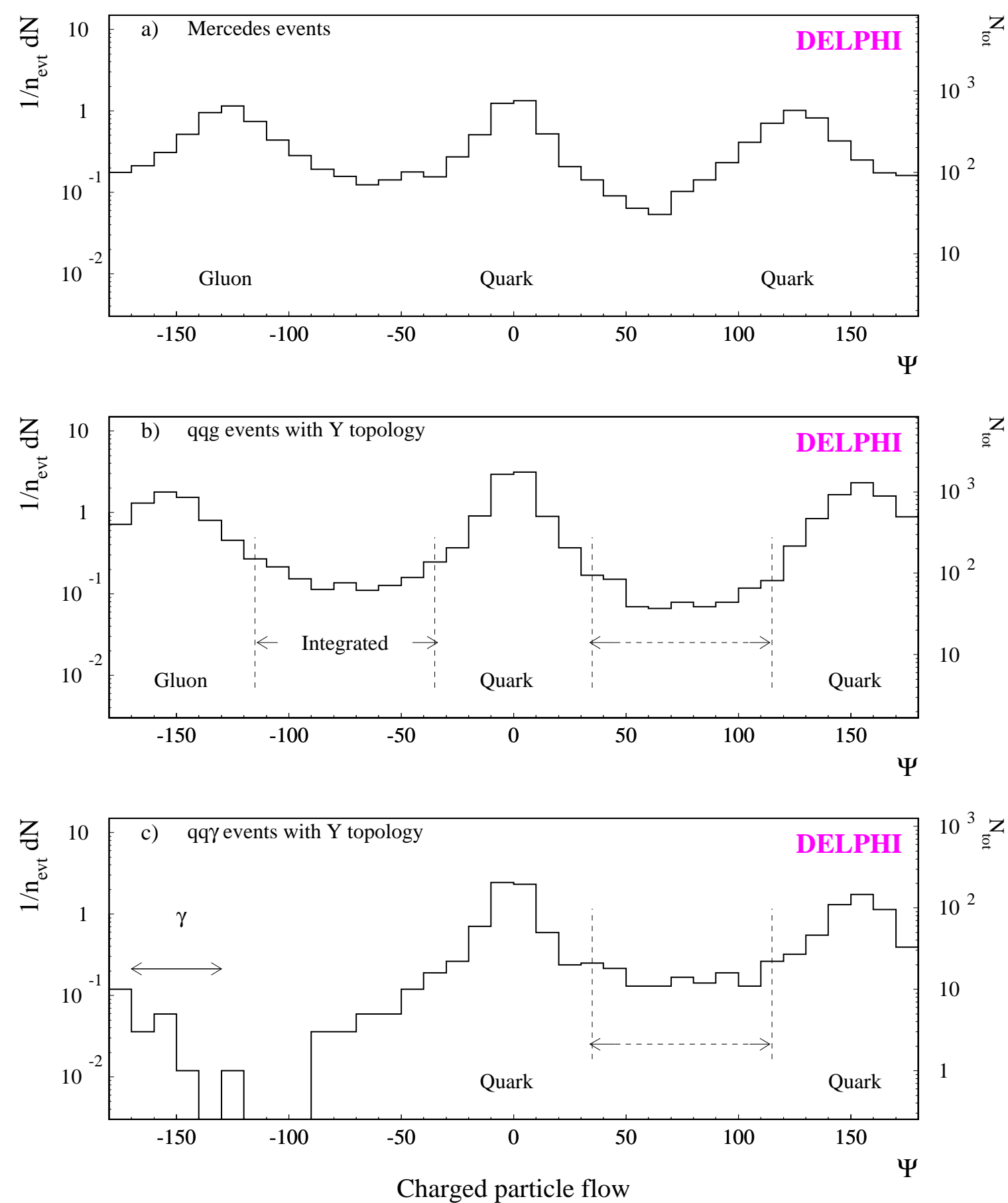

Figure 8: Charged particle flow for various three jet event configurations: (a) Mercedes $q \bar{q} g$ events, (b) Y $q \bar{q} g$ events and (c) Y $q \bar{q} \gamma$ events. In all plots, the number of particles is also indicated by the vertical scale at the right side. 
This result can then be compared to the asymptotic perturbative QCD expectation [5] which, for the specific topological configuration of this analysis, can be parametrised according to:

$$
R_{\gamma}(Q C D)=\frac{N_{q \bar{q}}(q \bar{q} g)}{N_{q \bar{q}}(q \bar{q} \gamma)} \approx \frac{0.65 n_{c}^{2}-1}{n_{c}^{2}-1} \sim 0.60,
$$

with $n_{c}=3$ representing the number of colours.

This result shows for the first time that inter-jet coherence can be measured to occur according to the perturbative QCD prescription.

In case the ratio $R_{\gamma}$ is calculated using $q \bar{q} g$ events with the leptonic tag its value increases to $R_{\gamma}(\mu, e)=0.68 \pm 0.07$ which is qualitatively in rather good agreement with $[9,10]$ taking into account that for each analysis the inclusive lepton selection is different. This result also confirms studies from $[8,9]$ which find that the measured interjet coherence effect slightly increases when the used quark and gluon jet samples contain higher purities. The above ratios are obtained directly from the measured data, but, if $R_{\gamma}(2 v t x)$ and $R_{\gamma}(\mu, e)$ were corrected according to our understanding of the sample purities and kinematic effects, corrections of $+3 \%$ for $R_{\gamma}(2 v t x)$ and $-19 \%$ for $R_{\gamma}(\mu, e)$ would be obtained. The combination of the corrected values of $R_{\gamma}$ using the results from various anti-tagged gluon samples looks attractive but has little effect in reducing the statistical error since this is limited by the number of events in the $q \bar{q} \gamma$ sample which is common for all sets, and, furthermore, the use of large correction factors may lessen the credibility of the result. If only the anti-tagged double vertex gluon sample is considered, the corrected value for $R_{\gamma}(2 v t x)$ is:

$$
R_{\gamma}(2 v t x)=0.58 \pm 0.06(\text { stat. }+ \text { syst. })
$$

\section{Charged Multiplicity in $\gamma+n-$ jet Events}

Scaling violations of the fragmentation functions of quarks and gluons are predicted in QCD [5]. The distribution of the scaled energy $x_{E}$ of the final state hadrons depends therefore on the center-of-mass energy $\sqrt{s}$. When the center-of-mass energy increases more phase space for gluon radiation becomes available leading to a softer spectrum of the scaled energy of the produced hadrons. The probability for gluon radiation is proportional to the strong coupling constant and hence it is possible to use QCD calculations in order to determine $\alpha_{s}$ when measuring such distributions at different center-of-mass energies [34]. As a consequence of these phenomena, the multiplicity of $e^{+} e^{-}$final state hadronic events also depends on the center-of-mass energy even though with opposite behaviour, as it increases when the average $x_{E}$ decreases. The QCD prediction for this latter observable has been computed as a function of $\alpha_{s}$ including the resummation of leading (LLA) and next-to-leading (NLLA) corrections [35]:

$$
n_{c h}(s)=a \alpha_{s}(s)^{b} e^{c / \sqrt{\alpha_{s}(s)}}\left[1+O\left(\sqrt{\alpha_{s}(s)}\right)\right]
$$

where $s$ is the squared center-of-mass energy and $a$ is a parameter not calculable from perturbation theory whose value has been fitted from data [35,36]. The constants $b=0.49$ and $c=2.27$ are predicted by the theory and $\alpha_{s}(s)$ is the strong coupling constant. This expression is, however, subject to sizeable corrections due to the neglected higher order terms, $O\left(\sqrt{\alpha_{s}(s)}\right)$. 
The emission of high energy radiative final state photons is well established and follows QED rules without interfering with the strong QCD processes involved in the hadronisation of quarks. It seems therefore reasonable to extract the photon from each $q \bar{q} \gamma$ event and study the corresponding mean charged multiplicity, $n_{c h}$, of the $q \bar{q}$ system as a function of its reduced squared center-of-mass energy: $s^{\prime}=s\left(1-2 E_{\gamma} / \sqrt{s}\right)$. It is then reasonable to compare the distribution thus obtained with the theoretical perturbative QCD calculation of Eq. 6. The result serves to cross-check the absence of biases in the quark jet sample used for the previous analyses and, furthermore, the value of $\alpha_{s}$ which can be fitted from Eq. 6 can indicate to which extend and under which circumstances this approach is valid.

Corrections due to the different flavour composition between the two processes of interest, $e^{+} e^{-} \rightarrow q \bar{q}$ at rather low $e^{+} e^{-}$energies and $e^{+} e^{-} \rightarrow q \bar{q} \gamma$ at $\sqrt{s} \approx M_{Z}$, should be, in principle, considered. At $\sqrt{s}<<M_{Z}$ the former interaction is mainly governed by the charge of the produced quarks that couple to the virtual intermediate photon. For the latter reaction, at LEP energies, the situation is a bit more complicated as the quark production is governed by the weak coupling of the quark to the $Z$ boson and, the final state photons are radiated according to the quark electric charge squared. The convolution of these two processes determines the flavour composition in this latter case which slightly differs from that obtained at lower $e^{+} e^{-}$energies, namely $+1.7 \%$ for $d$-type quarks and $-2.6 \%$ for $u$-type quarks. In the case of $b$ quarks, effective mass effects further reduced this difference by about $1.1 \%[37,38]$. In the least favourable case of $b$ quarks, which contain the largest mean charged multiplicity values, the different flavour composition due to the various center-of-mass energies at which the $e^{+} e^{-}$interaction takes place, produces shifts in the mean charged multiplicity distribution of less than 0.04 particles, according to the reported multiplicity difference of [29,30]. Therefore, this effect can be safely neglected.

In order to include all possibilities in which hard gluon radiation may occur before the emission of photons, other possibilities than just $\gamma+2-$ jets ought to be included. For this purpose, topologies with $\gamma+n-j e t s$ ( $n$ running from 1 to $\leq 4$ ) are also considered in this section. A total of 129 extra events are found to fulfil this condition in the DURHAM selected sample and are added to the $q \bar{q} \gamma$ sample whose selection is described in section 2.3.2. These data originate mainly from events with $n$ greater than two and populate the $s^{\prime}$ region close to $M_{Z}$.

The effect on the multiplicity distribution of requiring a minimum isolation angle for the photon of $20^{\circ}$ has also been studied and quantified. A small correction of the order of $1-2 \%$ has been considered depending on $s^{\prime}$ for the available kinematic energy range $s^{\prime} \leq 85 \mathrm{GeV}$.

The mean charged multiplicity of these events has been calculated using the unfolding method explained in section 5.1. As indicated in figure 9, a very good agreement is achieved when comparing these data to the resulting fits to data collected at lower $e^{+} e^{-}$ squared center-of-mass energies, $s \equiv s^{\prime}$, of references [35,36]. The parameters used have been: $a=0.059 \pm 0.012$ and $\alpha_{s}\left(M_{Z}\right)=0.106$, as derived in [36]. A further test can still be envisaged by comparing the mean charged multiplicity at $\sqrt{s}=M_{Z}$ as obtained from an independent DELPHI analysis [29] and the extrapolation of the present results. This is also shown in figure 9, where the data point corresponding to $\sqrt{s}=M_{Z}$ has been modified from that of [29] to account for the different flavour composition. A reasonably smooth transition can be observed in the curves. All these results confirm that the present analysis is firmly supported and consistent with previous measurements and thus that the quark sample extracted from $q \bar{q} \gamma$ events is unbiased. 


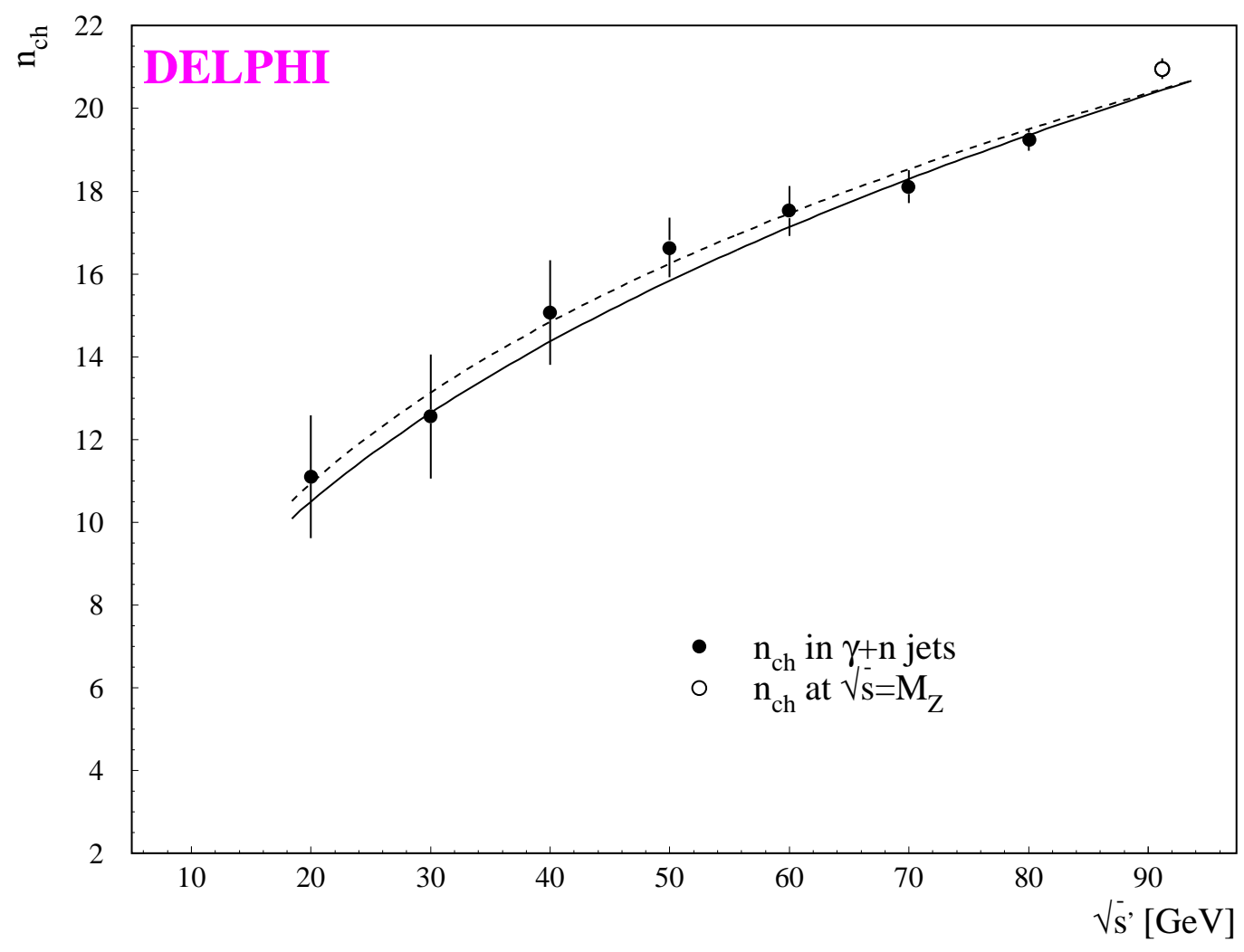

Figure 9: Charged multiplicity as a function of the effective center-of-mass energy $\sqrt{s^{\prime}}$. The value corresponding to $\sqrt{s^{\prime}} \approx M_{Z}$ is extracted from [29], notice however that only the statistical error is shown. The dashed and solid curves correspond to the fit from [36] and from our best fit using Eq. 7 including all data points, respectively.

A value for $\alpha_{s}\left(M_{Z}\right)$ can be extracted, using only DELPHI data, by directly fitting Eq. 6 to the mean charged multiplicity. Due to the limited statistics the uncertainty on $\alpha_{s}\left(M_{Z}\right)$ is large but can be reduced if a fixed value of the $a$ parameter is used. In order to consider the effect of the higher order corrections to Eq. 6 , an estimator $d$ can be introduced in the form:

$$
n_{c h}(s)=a \alpha_{s}(s)^{b} e^{c / \sqrt{\alpha_{s}(s)}}\left[1+d \cdot \sqrt{\alpha_{s}(s)}\right] .
$$

A fit to data using Eq. 7 with $\alpha_{s}\left(M_{Z}\right)$, being expressed at second order, and $d$ as free parameters leads to the following result:

$$
\alpha_{s}\left(M_{Z}\right)=0.114 \pm 0.005 \text { (stat.) } \pm 0.008 \text { (syst. : a) } \pm 0.005 \text { (theo. : d) },
$$

where the first systematic error indicates the dependence w.r.t. the value of the parameter $a$ and the second is due to the $d$ parameter whose fitted value is $0.58 \pm 0.47$.

In case all data are used in the fit, i.e. including the last energy point at $\sqrt{s}=M_{Z}$ with systematic and statistical errors being considered, the increase in statistics enables 
the three parameters, $a, d$ and $\alpha_{s}(s)$, to be adjusted at the same time. The result is:

$$
\left.\alpha_{s}\left(M_{Z}\right)=0.116 \pm 0.003 \text { (stat.) } \pm 0.007 \text { (syst. : pur.) } \pm 0.005 \text { (theo. : } d\right) \text {, }
$$

with $a=0.070 \pm 0.015$ and $d=0.20 \pm 0.32$. The first systematic error has been computed taking into account the effect of the purity correction when adapting the last energy point at $\sqrt{s}=M_{Z}$ from reference [29] to the present analysis. Note, however, the large existing correlation factor of $\sim 0.3$ between the parameters $a$ and $\alpha_{s}(s)$, since the former acts as kind of "offset" and the latter determines the "slope". The reduction of this dependence therefore requires more data to be used in the fit, especially at the lowest energy points. The obtained $\chi^{2} / n . d . f$. in this fit is 0.9 .

\section{Summary and Conclusion}

Properties of quark and gluon jets are deduced from $Z$ hadronic decays into three jets which have been identified as $q \bar{q} g$ and $q \bar{q} \gamma$ final states. Heavy quark tagging by the vertex detector and lepton identification has been used to anti-tag the gluon jets. These jets are compared to quark jets of similar energies measured in $q \bar{q} \gamma$ events or quark and gluon jet mixtures in symmetric three jet events. The extracted properties of pure quark and gluon jets do not depend on corrections using fragmentation models and are insensitive to the $b$ quark fragmentation because the quark reference samples are depleted from $b$ events.

The average ratio of the mean charged multiplicity in gluon and quark jets specified with the DURHAM algorithm is found to be

$$
<r>=1.241 \pm 0.015 \text { (stat.) } \pm 0.025 \text { (syst.) }
$$

This result is obtained using three different techniques which yield consistent results.

The data exhibit an energy dependence of the ratio $r$ which was fitted using a linear function to yield the slope

$$
\Delta r / \Delta E=(86 \pm 29 \text { (stat.) } \pm 14 \text { (syst.) }) \cdot 10^{-4} \mathrm{GeV}^{-1} .
$$

The indication for the energy dependence $(2.7 \sigma)$ comes mainly from the comparison of $q \bar{q} g$ and $q \bar{q} \gamma$ events but is supported by the studies of symmetric events.

Similar energy behaviour is found when the JADE algorithm is used, even though with a significant offset of $r$ towards a higher value

$$
<r>=1.369 \pm 0.019 \text { (stat.) } \pm 0.035 \text { (syst.) }
$$

indicating that for a given fixed energy there is still a further dependence on the value of $r$ due to the algorithm used to reconstructed jets, mostly correlated with its intrinsic angular acceptance for soft particles [19]. As a consequence of this and as could be expected from the gluon particle spectrum, gluon jet multiplicities have been measured to be more sensitive to this effect than the corresponding multiplicities from quark jets.

The inclusive particle spectrum from gluon jets is found to be much softer compared to quark jets. At large momenta the cross section for gluons is suppressed by almost one order of magnitude. The increase in multiplicity takes place at small momentum. A slight decrease of the inclusive spectra at large $x_{E}$ with energy for quark and gluon jets is observed, being stronger for gluon jets. For quark jets it is consistent with the energy 
dependence measured by lower energy experiments attributed to gluon radiation (scaling violations). In the case of gluons a stronger energy dependence is measured as expected because of the higher radiation probability for gluons due to their higher colour charge.

The softer spectrum of gluon jets is supported by the study of the leading particle rapidity. The energy dependence and quark gluon difference are consistent with expectations. Gluon jets are observed to be broader than quark jets.

Studies of the string effect show that it is present in fully symmetric events and, also, that it depends on the colour nature of the initial three jet configuration. A quantitative comparison of the particle rates in the region opposite to the gluon and to the photon in $q \bar{q} g$ and $q \bar{q} \gamma$ events yields

$$
R_{\gamma}(2 v t x)=0.58 \pm 0.06(\text { stat. }+ \text { syst. })
$$

consistent with the asymptotic perturbative QCD expectation for this topology, $R_{Q C D} \simeq 0.6$. This result confirms for the first time that inter-jet coherence measurements agree with the quantitative perturbative QCD prescription.

The validity of this result is underlined by the study of the mean charged multiplicity in $q \bar{q} \gamma$ events. This is shown to follow nicely the expectation from lower energy $e^{+} e^{-}$data supporting our premise that the $q \bar{q} \gamma$ events are a relevant unbiased reference sample. A fit using the leading and next-to-leading order calculation to these data gives a value corresponding to the strong coupling constant $\alpha_{s}\left(M_{Z}\right)$ of

$$
\alpha_{s}\left(M_{Z}\right)=0.116 \pm 0.003 \text { (stat.) } \pm 0.009 \text { (syst.) }
$$

which is in good agreement with other measurements of $\alpha_{s}$, especially with the fit to the data on charged multiplicity at lower center-of-mass energies [35,36].

\section{Acknowledgements}

We are greatly indebted to our technical collaborators and to the funding agencies for their support in building and operating the DELPHI detector, and to the members of the CERN-SL Division for the excellent performance of the LEP collider. We also thank A. Giovannini, M. Kelsey, A. Pich, A. Santamaria and M. Seymour for useful and illuminating discussions. 


\section{References}

[1] JADE Coll., W. Bartel et al., Z. Phys. C21 (1983) 37;

JADE Coll., W. Bartel et al., Phys. Lett. B123 (1983) 460;

UA2 Coll., P. Bagnaia et al., Phys. Lett. B144 (1984) 291;

MARK II Coll., A. Peterson et al., Phys. Rev. Lett. 55 (1985) 1954;

HRS Coll., M. Derrick et al., Phys. Lett. B165 (1985) 449;

UA1 Coll., G. Arnison et al., Nucl. Phys. B276 (1986) 253;

AMY Coll., Y.K. Kim et al., Phys. Rev. Lett. 63 (1989) 1772;

TASSO Coll., W. Braunschweig et al., Z. Phys. C45 (1989) 1;

AMY Coll., M. Ye et al., Proc. 25th Int. Conf. on High Energy Physics, August 1990, Singapore, Vol. II, Eds. K.K. Phua, Y. Yamaguchi, Pub. South East Asia

Theoretical Physics Association and the Physical Society of Japan (1991) p. 889;

DELPHI Coll., P. Abreu et al., Z. Phys. C56 (1992) 63;

CLEO Coll., M.S. Alam et al., Phys. Rev. D46 (1992) 4822;

TOPAZ Coll., Y. Ohnishi et al., KEK preprint 93-73 (1993), to be published in Phys. Lett. B;

VENUS Coll., H. Takaki et al., Phys. Rev. Lett. 71 (1993) 38.

[2] OPAL Coll., G. Alexander et al., Phys. Lett. B265 (1991) 462;

OPAL Coll., P.D. Acton et al., Z. Phys. C58 (1993) 387;

OPAL Coll., R. Akers et al., CERN-PPE/95-075.

[3] ALEPH Coll., D. Busculic et al., Phys. Lett. B346 (1995) 389.

[4] M. L. Mangano, P. Nason, Phys. Lett. B285 (1992) 160;

M. H. Seymour, Z. Phys. C63 (1994) 99;

M. H. Seymour, Nucl. Phys. B436 (1995) 163.

[5] Y.L. Dokshitzer, V.A. Khoze, A.H. Mueller, S.I. Troyan, "Basics of perturbative QCD", Editions Frontiers, 1991.

[6] Ya.I. Azimov, Yu.L. Dokshitzer, V.A. Khoze, S.I. Troyan, Phys. Lett. B165 (1985) 147 ;

Ya.I. Azimov, Yu.L. Dokshitzer, V.A. Khoze, S.I. Troyan, Yad. Fiz. 43 (1986) 149. T. Sjöstrand, ' $Z^{0}$ Physics', Cargèse 1990, Eds. M. Lévy et al., Pub. Plenum Press, New York (1991) 367; CERN-TH.5902/90 "QCD and Jets at LEP".

[7] TPC Coll., H. Aihara et al.: Phys. Rev. Lett. 57 (1986) 945;

MARK II Coll., P.D. Seldon et al.: Phys. Rev. Lett. 57 (1986) 1398;

JADE Coll., F. Ould Saada et al.: Z. Phys. C39 (1988) 1.

[8] OPAL Coll., M. Z. Akrawy et al., Phys. Lett. B261 (1991) 334;

OPAL Coll., R. Akers et al., CERN-PPE/95-83.

[9] L3 Coll., M. Acciarri et al., Phys. Lett. B345 (1995) 93-102;

D. Duchesneau, Ph. D. Thesis, "Etude du rayonnement électromagnétique des quarks primaires produits dans les désintégrations hadroniques du Z avec le détecteur L3 au LEP", Thèse No. 2644, Université de Genève 1993.

[10] ALEPH Coll., D. Buskulic et al., Contribution to the 27th International Conference on High Energy Physics, Glasgow, Scotland, July, 1994. ICHEP94 ref. 0543 .

[11] DELPHI Coll., P. Aarnio et al., Nucl. Instr. Meth. A303 (1991) 233.

[12] DELPHI Trigger Group (V. Bocci, et al.), Architecture and Performance of the Delphi Trigger System, Nucl. Instr. Meth. A362 (1995) 361.

[13] W. Adam et al., Design and Performance of the Delphi Data Acquisition System, IEEE Trans. Nucl. Sci. 39 (1992) 166. 
[14] N. Bingefors et al., Nucl. Instr. Meth. A328 (1993) 447.

[15] DELPHI Coll., P. Abreu et al., Phys. Lett. B307 (1993) 221.

[16] S. Bethke, Z. Kunszt, D. Soper, W..J. Stirling, Nucl. Phys. B370 (1992) 310.

[17] S. Catani et al., Phys. Lett. B269 (1991) 432;

N. Brown, W.J. Stirling, Z. Phys. C53 (1992) 629.

[18] JADE Coll., W. Bartel et al., Z. Phys. C33 (1986) 23;

JADE Coll., S. Bethke et al., Phys. Lett. B213 (1988) 235.

[19] P.V. Chliapnikov, V.A Uvarov, F. Bianchi, Phys. Lett. B300 (1993) 183.

[20] S. Martí Ph. D. Thesis, "Estudi de la dependència energética de les diferències entre jets de quarks i de gluons utilitzant el detector DELPHI de LEP". Universitat de València, IFIC/95-59.

[21] O. Klapp, Diplomarbeit "Quark- und Gluonjets aus Z-Zerfällen — Vergleichende Studien des Jetendzustandes und der Substruktur". WU D 95-15. University of Wuppertal.

[22] T. Sjöstrand, Comp. Phys. Comm. 39 (1986) 346;

T. Sjöstrand, M. Bengtsson, Comp. Phys. Comm. 43 (1987) 367.

[23] DELSIM user's guide, DELPHI 89-15 PROG 130, CERN, February 1989;

DELSIM Reference Manual, DELPHI 89-68 PROG 143, CERN, September 1989.

[24] DELPHI Coll., P. Aarnio et al., Phys. Lett. B341 (1994) 109.

[25] ALEPH Coll., D. Buskulic et al., Phys. Lett. B313 (1993) 535.

[26] G.V. Borisov, DELPHI Internal Note, DELPHI 94-27 PHYS 361 (1994);

G.V. Borisov, Lifetime Tag of Events with B-hadrons with the DELPHI detector, preprint IHEP (Protvino), 94-98 (1994);

DELPHI Coll., P. Abreu et al., Z. Phys. C65 (1995) 555.

[27] See for example A.S. Schwarz, Phys. Rep. 238 (1994) 1, and references therein.

[28] F. Bianchi, P.V. Chliapnikov, L. Gerdyukov, A. Giovannini, "A study of the ratio of quark and gluon jet mean charged particle multiplicity at LEP by the correlation method", DELPHI 95-40 PHYS 483.

[29] DELPHI Coll., P. Abreu et al., Phys. Lett. B347 (1995) 447.

[30] OPAL Coll., R. Akers et al., CERN-PPE/95-038.

[31] Z. Fodor, Phys. Lett. B263 (1991) 305.

[32] AMY Coll., Y.K. Li et al., Phys. Rev. D41 (1990) 2675.

[33] TASSO Coll., W. Braunschweig et al., Z. Phys. C47 (1990) 187.

[34] DELPHI Coll., P.Abreu et al., Phys. Lett. B311 (1993) 408;

ALEPH Coll., D. Busculic et al., CERN-PPE/95-096.

[35] B.R. Weber, Phys. Lett. B143 (1984) 501 and references therein.

[36] P.V. Chliapnikov, V.A. Uvarov, Phys. Lett. B251 (1990) 192 and references therein.

[37] A. Ballestrero, E. Maina and S. Moretti, Phys. Lett. B294 (1992) 425.

[38] M. Bilenky, G. Rodrigo, A. Santamaria, Nucl. Phys. B439 (1995) 505. 